\title{
The impact of increased decentralised generation on the reliability of an existing electricity network
}

\author{
Anton Johannes Veldhuis ${ }^{1}$, Matthew Leach ${ }^{2}$, Aidong Yang ${ }^{1}$ \\ 1. Department of Engineering Science, University of Oxford \\ 2. Centre for Environment and Sustainability, University of Surrey
}

\begin{abstract}
This study evaluates the impact of decentralisation on the reliability of electricity networks, particularly under stressed conditions. By applying four strategies to add decentralised generators to the grid, the impact on network reliability has been assessed, where the blackout impact has been defined as the product of the relative blackout size and the relative blackout frequency. The general approach taken to decentralise the network is to replace the aggregated generation capacity at an existing node with three new nodes representing the total generation capacity of multiple decentralised generators. Two different networks have been used: a reduced and aggregated version of the electricity network of Great Britain (GB) and the IEEE 39 network, and each of them has been assessed for decentralisation based on conventional energy sources and for decentralisation based on intermittent renewable energy sources. The results suggest that adding significant amounts of DGs, especially if it is intermittent, can seriously reduce network reliability; however, various approaches regarding the decentralisation strategy and management of the resulting network can mitigate the negative effects.
\end{abstract}

Keywords: power networks, decentralised generation, reliability, simulation

\section{Introduction}

The future of electricity generation will become more and more decentralised. This is primarily due to the increasing amount of wind and solar energy systems, supplemented by the installation of other smaller-scale generation facilities (e.g. hydro, anaerobic digestion and combined heat and power (CHP) systems) [1]. Since the decentralisation changes the traditional centralised power supply, the network operators tend to have less control over the power generators. Moreover, the existing electricity network will be used in a different way than was envisaged during its original design, since surplus power in a distribution grid might reverse the direction of power flow. In general, the grid is required to become more flexible in order to balance the supply and demand $[2,3]$.

It is well-known that a national electricity system consists of multiple networks with different voltage levels which are interconnected by transformers; in the UK, these levels include a high voltage (HV) transmission network (400kV, $275 \mathrm{kV}$ and $132 \mathrm{kV}$ ), a medium voltage (MV) distribution network (33kV and $11 \mathrm{kV}$ ) and a low voltage (LV) distribution network (230V). DGs directly connected to the distribution grid such as rooftop PV are defined as distributed generators [4]. Distributed generators will mainly result in a lower electricity demand in the HV grid, since part of the demand will be met by these local generators [5]. However, under the influence of cost reductions in renewable power generation technologies combined with policies favouring low carbon generation, the share of distributed energy systems will likely increase further. With higher shares of distributed energy 
systems, it becomes more likely that a surplus of power generated in a distribution grid will be transferred to the MV and eventually the HV grid.

The Paris agreement aims to keep the "global temperature rise this century well below 2 Celsius above pre-industrial levels and to pursue efforts to limit the temperature increase even further to 1.5 degrees Celsius" [6]. To meet this goal, it is likely that governments will decide to develop more large-scale energy projects such as offshore wind farms. These (grouped) DGs will be connected to the HV grid, which will change the power flow distribution in the HV grid. Eventually, current fossil fuel based power plants will be phased out. The combination of distributed energy systems, decentralized energy systems and the phasing out of existing fossil fuel based generators will lead to different load profiles of the HV grid. For distributed energy systems like rooftop PV, it might be hard to plan and control the locations of the installations, unless the regulatory practice changes dramatically in order to cope significant network management challenges. For large scale energy projects such as wind farms, the locations can be more readily planned. It is therefore interesting to know what the impact of the locations of new DGs will be on the reliability of the grid, so that system planners could take this into account.

In this study, we are interested in the impact of the above future changes on the reliability of the power supply of a nationwide system. Consequently, we will focus on the HV transmission grid to evaluate the impact of introducing DGs, through changing the level (and, in the case of renewable DGs, the temporal pattern) of supply at the affected transmission nodes, on the reliability of the existing network structure.

For electricity grid operators, the reliability of the network, which relates to the security of supply for customers, is one of the most critical concerns. Since society depends increasingly on electricity, power outages should be minimised in order to avoid major disruptions to normal daily life potentially leading to high societal costs [7-9]. The main objective of network operators is to ensure a reliable electricity supply while minimizing the operating costs.

Network operators use economic dispatch (ED) models to minimize the operating costs of a network subject to network constraints. To account for risks of failing network components, such as generator and line outages, the system operators extend the ED models by including additional security constraints [10]. These models are known as security-constrained economic dispatch models (SCED) [10-13]. Typically, network operators will make sure that a network satisfies the N-1 condition, meaning that uninterrupted electricity supply is still guaranteed if a single network component fails $[10,14,15]$. To improve the network, network operators have multiple ways to address reliability issues. One of these is transmission expansion planning (TEP) [16], which studies which transmission lines should be upgraded and where it makes sense to create extra lines. For example, the inclusion of wind power in the grid has to be taken into account in TEP $[17,18]$.

Historically, the HV grid has been very reliable due to safeguards such as the $\mathrm{N}-1$ condition and capacity margins in both generation and line capacities. Currently, the reliability of the electricity networks in Western Europe are very high and blackouts are therefore very unlikely, for example the reliability of UK's national grid has been 99.999998\% during the financial year 2015/16 [19]. However, future developments and changes such as the decentralisation of electricity supply might change load profiles leading to different stresses in the network. At the same time, highly unlikely events do occur, in November 2016 a boat's anchor caused serious damage to the France-UK 
interconnection cables, which typically supply about $5 \%$ of UK's electricity [20]. Likewise, accidents with defense helicopters hitting transmission cables are rare, but have happened occasionally [21]. If such events happen when the electricity system is already strained by high inputs of renewables, this might lead to large blackouts.

A lot of research has been focused on the reliability, vulnerability or robustness of a power network. A study by Koç et al. [22] presents a metric to quantify the network robustness with respect to cascading failures. The metric takes both flow dynamics and network topology into account. In another paper, the same authors use the effective graph resistance as a metric to assess the robustness of a network topology against cascading failures by targeted attacks [23].

Few studies use simulations to quantify this impact. On the connection between decentralisation and network stability, Rohden et al. [24] studied the self-organized synchronization of the nonlinear dynamics of complex power networks with increasing decentralisation of the British power grid, and found that more decentralized grids may have moderately lower dynamic stability, but that they become more robust to structural failures at the same time due to the increased self-organised synchrony. Zerriffi et al. [25] evaluated network robustness and costs of centralised and distributed gas and electric power distribution systems for different failure modes. They applied a modified IEEE reliability test system and evaluated the availability of the individual generating units as a key variable in their Monte Carlo simulation, with the loss of energy expectation as the metric to assess the reliability of various networks. They found that electric power systems with DGs improve reliability. However, they assumed that power lines in the central part of the grid experience no line outages and their approach did not include power flow dynamics and thus no cascading failures.

To assess blackout dynamics in power networks, Carreras et al. [26] developed the ORNL-PSercAlaska (OPA) model. Using the well-known DC power flow model, the core of the simulation includes slow and fast dynamics. During the slow dynamics, the energy demand and generation capacity will be increased by a small amount. The fast timescale represents the actual operation of the network, particularly the power line outages by relay protection ${ }^{1}$ which includes some probability of operating incorrectly, redistribution in flows following outage and subsequent further line outages (a phenomenon known as the cascading effect). The authors examined the sensitivity of several network structures by varying model parameters. They found that "although the size of the network affects the sizes of the cascading events, it appears to have little impact on the frequency of the events" [26].

Mei et al. [27] adapted the OPA model of Carreras et al. by introducing two changes to be consistent with practical systems, namely decreasing the "tripping" probability of an overloaded line, and including a planning function inside the slow dynamics part of the simulation. This planning function increases the line capacities of overloaded lines based on an initial power flow simulation before the actual power flow will be simulated.

Focusing on the impact of increasing decentralisation on power network reliability, this study is designed to quantify, through simulation studies, the blackout behaviour for electricity networks with different levels of decentralisation considering both power flow dynamics and network

\footnotetext{
${ }^{1}$ Power lines are protected by relays which can trip a circuit breaker when a fault is detected (e.g. over-current or over-voltage).
} 
structural features, which has not been carried out before. The novelty of this study is to apply an existing power flow model - which has been developed to analyse blackout dynamics in a grid - to an electricity network which will be decentralised incrementally, in order to analyse the reliability of decentralisation. By applying different strategies, the results can give insights on the best approaches to increase the reliability of a highly decentralised power network. In particular, reliability in this study is quantified by the blackout size and frequency, with a focus on line failures, not on generator failures.

Note that in a real system, the risks of the same blackout frequency and size can be different, because of dissimilar economic or social ties related to specific nodes [28], however, this study simplifies some of these real-world complexities, to allow focus on a set of key technical attributes of the decentralisation process.

This paper is organised as follows. The next section will present the methodology, which starts with a general description of electricity grids and a more specific description of the two applied electricity networks. Subsequently, the power flow model and the modelling of DGs will be discussed. The latter will distinguish two cases: (1) conventional DGs and (2) renewable DGs. The results are presented in section 3 , which is followed by a discussion section. The paper concludes with the main findings and potential areas for future work.

\section{Methodology}

In this study, a HV power grid will be modelled as a graph to analyse the blackouts caused by cascading effects due to line overloads. By incrementally increasing the share of DGs in the network, we will simulate annual power demand and supply profiles through calculating the power flows for two types of DGs: DGs based on conventionally fuelled generators with a controllable power output and DGs based on renewables with an intermittent power output. For the former we will apply a time step of a day with a fixed power demand; for the latter, we will use an hourly time interval with an annual demand profile. The output of the renewables will be based on weather data of an exemplar location from a single year. Two case studies will be carried out by applying the model to two different networks. By analysing the results, we will be able to see whether these blackouts change in size and frequency while increasing the penetration level of DGs as a measure for the impact of decentralisation on the reliability of a HV grid.

By focusing on the HV grid, we assume that the nodes representing aggregated distribution grids have the capacity to cope with any issues or "noise" in the distribution networks caused by the additions of DGs, hence the details at the distribution network level are not considered in this work.

To model a power grid as a graph, generation, transmission, distribution buses and transformers are represented by nodes. The transmission lines are modelled as links. The following sub sections will discuss the model in more detail.

\subsection{Power flow model}

The power flow in a HV grid is based on alternating current (AC) and it is controlled by the grid's impedances, voltage levels at each individual power station, voltage phase differences between power stations and loads at terminal stations [22]. To estimate the power flow values for each component in the grid, power flow equations can be used. For AC power, non-linear equations are 
used to model both the active and reactive powers. Direct current (DC) power flow equations are a simplification and linearization of the $A C$ equations and consider the active power flows only. The following assumptions have been made in order to get the DC power flow equations [22]:

- Reactive power balance equations are neglected;

- All voltage magnitudes are considered to be $1 p . u$.;

- Line resistance is ignored so that line impedance equals line reactance;

- Voltage phase differences are very small so that $\sin \theta_{i j} \approx \theta_{i j}$, where $\theta_{i j}$ is the voltage phase difference between node $i$ and node $j$

The usefulness of the DC power flow model for active power flow analysis has been studied by Purchala et al. [29] by comparing the results with the AC power flow. Based on various randomly generated networks with changing topology, size and system load, they found that most errors are within the range of $5 \%$, although errors on individual lines can occasionally be significant.

Furthermore, line resistances are neglected in the DC power flow model, therefore it should only be applied to high voltage transmission grids. Since we focus on the HV power grid, the DC power flow model can be applied to estimate the flow values across the network.

The improved OPA model from Mei et al. [27] is used as a basis for this study, but with changes to the so-called slow dynamics to put focus on the modelling of the blackout behaviour due to decentralisation rather than increased electricity demand. Therefore, the model in this study will neither increase the electricity demand nor include scheduled line improvements. Therefore, the electricity network will remain the same during the simulations, except for potential line outages. Any line outages will be restored at the beginning of a new day. The model has been implemented in MATLAB by extending the MATPOWER package version 6.0b1 [30].

Figure 1 shows a schematic view of the power flow procedure. Before the fast dynamics start, a random line outage can occur. Every original line in the network has an outage probability of $\tau$ representing accidental faults. With or without any accidental faults actually occurring, the DC power flow model always calculates the power flow in the network. When line flow limits or generation capacity limits are exceeded, the optimal power flow will be calculated. This is carried out by a dispatching centre which itself is subject to random failure leading to disruptions to its functioning. The failure rate of the dispatching centre is denoted by $\eta$, which is the probability that the optimization program is not run. In this case, some lines might get tripped which in turn can lead to multiple groups of nodes (i.e. islands). Potential isolated nodes are not taken into account in the further power flow run(s) for the specific time step. In such situation, if the isolated node is a generator, the generator capacity will become unavailable in the network. If the node has a (net) load, it is assumed that this load will not be served. When lines have been tripped, the network flows will be recalculated and the sub procedures will be repeated until a stable network has been found. Otherwise, when no lines have been tripped, the optimal power flow (OPF) will be executed to ensure that the power outputs of all generation nodes (including DGs) meet the network constraints and, in the case with renewable-based DGs, that any excess will be stored.

Every group of nodes needs a reference bus (i.e. voltage angle set to zero) in order to run the DC power flow model. If a group of nodes does not contain a reference bus, one of the nodes has to become one. The reference bus must be a generator node. Since the reference bus itself will be 
excluded from the linear equations in order to get a free variable, there should be at least another generator bus plus another node with a load to enable the calculation of the power flows. If this is not the case (e.g. a group of only two nodes), the possible load within this group will be modelled as a blackout, i.e. the demand cannot be met.

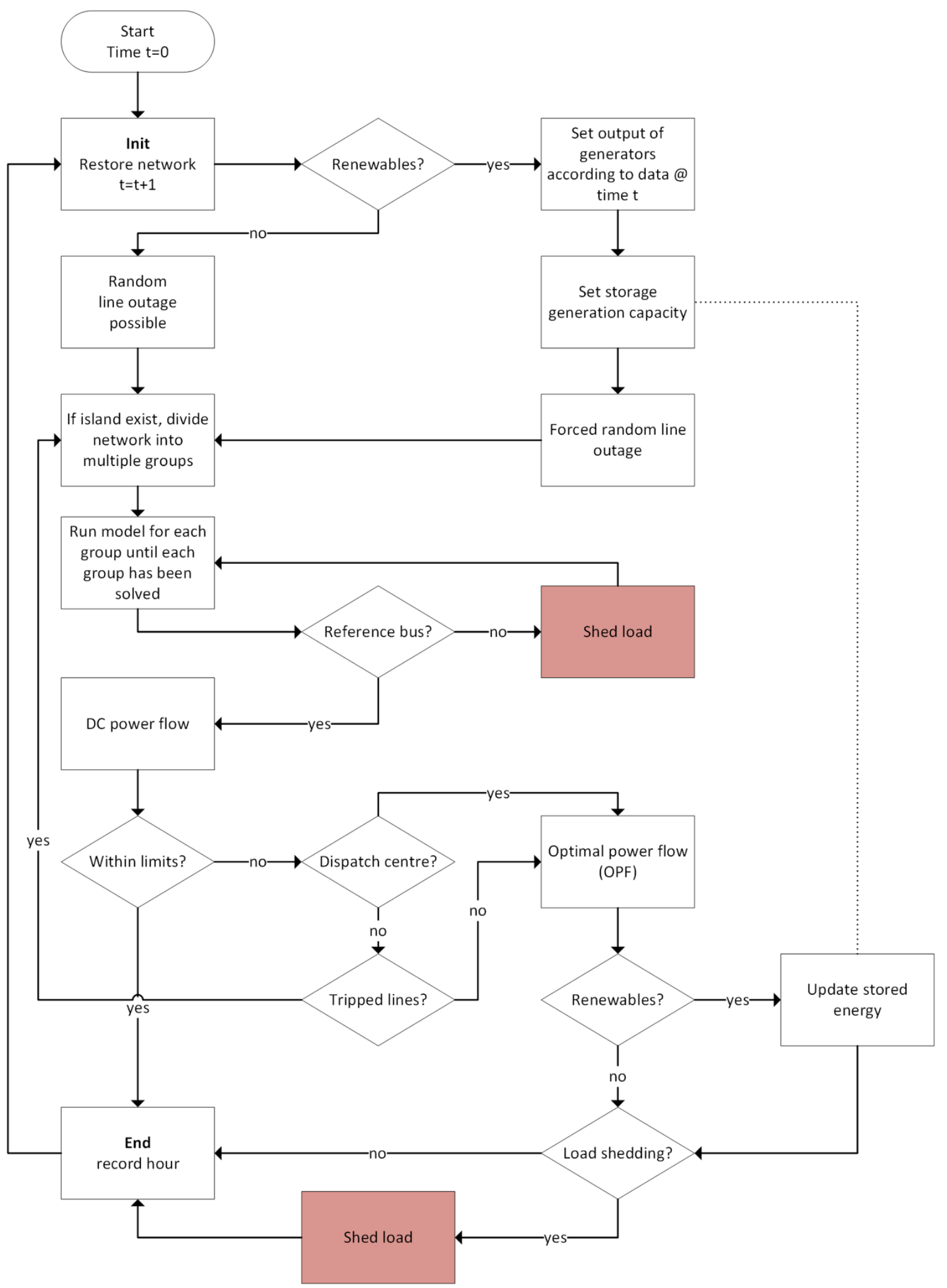

Figure 1: Schematic diagram showing the power flow model as applied in this study. 
The various probability parameters and their values adopted in this work are identical to those used in the improved OPA model [27]. The probability of tripping overloaded lines is set to $b$ (i.e. the normal functioning of relay protection). The probability of tripping normal lines is $\xi \times\left|F / F^{\max }\right|^{n}$, where $\xi$ is the base probability of unwanted operation of relay protection, $\left|F / F^{\max }\right|$ is the load ratio (between the actual and maximum flow, $\mathrm{F}$ and $\mathrm{F}^{\max }$ ) of the transmission line, and $n$ is the exponent. The values for the model parameters are shown in Table 1.

Table 1: Initial values for the parameters as applied in the improved OPA model

\begin{tabular}{|l|l|l|}
\hline \multicolumn{1}{|c|}{ Parameter description } & Symbol & \multicolumn{1}{c|}{ Value } \\
\hline The probability of accident & $\tau$ & 0.0007 \\
\hline The failure rate of the dispatching centre & $\eta$ & 0.05 \\
\hline The probability of tripping overloaded lines & 6 & 0.999 \\
\hline $\begin{array}{l}\text { The base probability of unwanted operation of relay } \\
\text { protection }\end{array}$ & $\xi$ & 0.001 \\
\hline Exponent normal tripping lines & $n$ & 10 \\
\hline
\end{tabular}

In this study, load shedding is regarded as the origin of a possible blackout. A blackout is defined as an event in which load shedding is larger than $10^{-5}$ times the total power demand, as also applied in [26]. Blackouts mainly occur during peak load when the network operates close to its critical point. Since we are interested in the impact of decentralisation on blackouts, we will simulate stressed networks.

\subsection{Quantifying reliability}

Reliability in the electricity sector can be quantified in various ways [31-34]. Reliability is defined in terms of two components: adequacy and security and can be generally described as "the ability to supply the quantity and quality of energy desired by the customer when it is needed" [31]. In general, outage frequency, duration and size are important measures. Because we use discrete time, the exact outage duration is outside the scope of this study. Hence, in this study, the two normalised main measures are the blackout size relative to the total load and the frequency of blackouts relative to the number of simulations. Since the blackout size and the blackout frequency alone could give a distorted view, another measure which combines both has been used: the blackout size multiplied by its relative frequency can be perceived as the blackout impact.

Additionally, the notion of power capacity imbalance $(\mathrm{Cl})$ is introduced in the analysis, which, always taking a non-negative value and calculated for a node, is defined as the difference between the generation capacity and the load of the node itself plus its direct neighbouring nodes.

The $\mathrm{Cl}$ of a node offers an indication of to what extent a node needs to exchange power flow with any neighbouring nodes of the network. As power flow exchange is physically implemented through power lines, the summation of the $\mathrm{Cls}$ of each node in a network thus reflects to a certain extent its vulnerability to line outage derived blackout.

\subsection{Modelling decentralised generators}

There are various ways to model a network with increased decentralised power generation. Most newly added decentralised power generators are renewable energy generators such as wind and solar energy systems. These renewable energy resources are intermittent and therefore cannot be 
modelled as conventional power generators which - within certain limits - can produce power on demand. To study separately the effect of decentralisation and that of temporal fluctuation in supply (and demand), the first part of this study treats DGs as conventional power generators with a steady dispatchable capacity such as CHP [35] and within a network for meeting a constant total demand. The second part will take into account the variable availability of capacity of the added DGs as well as a dynamic demand profile. In both parts, the intention is to explore the effects of greater decentralisation on the reliability of the network; therefore, we keep the overall supply capacity constant while introducing DGs to replace existing generators.

Since we are focusing on the HV grid, a DG node represents the aggregation of a group of smaller generators, for example a wind farm or a solar energy park.

\subsubsection{Part I: Systems with steady-capacity DGs and a constant demand}

To decentralise the network while maintaining constant overall supply, the total generation capacity of an original node in the network will be replaced by three newly added generators - each with a third of the replaced capacity - which will be connected to other locations in the network.

In reality, newly added DGs will not replace an existing generation capacity immediately, however, in the long run, when the share of DGs increases, the legacy capacity is expected be eventually removed.

This study compares the effect of random with planned decentralisation. Based on regional statistics [36] and common knowledge, it seems reasonable to assume that the introduction of most of the distributed generators such as rooftop PV and single wind turbines will more closely correspond to a random approach. Of course, the available energy resource (i.e. annual irradiation or average annual wind speeds) will play a role and so might particular local subsidy schemes; the majority of the locations of these distributed generators has not been planned by the network operator. To determine which generator will get replaced and where the new DGs will be added to the existing network, a completely random approach plus three alternative strategies will be applied, see Table

2. It can be seen that strategies 2 and 3 select the generator to be replaced based on the smallest generator impact. This generator impact is determined by calculating the blackout size for each generator/bus combination in the network, in which the capacity of the generator is distributed over three newly added DGs which are all connected to the same selected bus. Two power flow model runs (cf. Figure 2) are calculated, one with and another without activating the dispatching centre, both excluding random line outages. For each generator, the average blackout size is calculated based on these two runs for each possible bus for placing DGs; the generator placement with the smallest average blackout size is considered as one with the lowest "generator impact". If there are multiple generator placements with an equal impact, the generator placement which causes the lowest average $\mathrm{Cl}$ will be selected.

On the selection of the location for adding DGs, strategies aiming to reduce power capacity imbalance, hypothesised to potentially enhance network reliability, as well as random selection are tested. Note that both power imbalances and generator impacts are continuously updated while the network evolves with the addition of DGs.

Once the location for adding a DG is determined, a new power line is introduced and the line capacity is determined based on the capacity of the DG, the line reactance is set to the mean of the 
line reactances in the original network to avoid possible effects caused by using more extreme values and thus to focus on the location of the DGs only.

Table 2: Different strategies for introducing DGs.

\begin{tabular}{|l|l|l|}
\hline $\begin{array}{l}\text { Location } \\
\text { for adding DG }\end{array}$ & Choosing randortorly to replace & $\begin{array}{l}\text { Choosing one with } \\
\text { smallest } \\
\text { generator impact }\end{array}$ \\
\hline Choosing randomly & Random & Strategy 2 \\
\hline $\begin{array}{l}\text { Choosing node with largest power capacity } \\
\text { imbalance, incl. neighbouring nodes }\end{array}$ & Strategy 1 & Strategy 3 \\
\hline
\end{tabular}

The simulation study carried out for Part I is summarised as follows. Each strategy starts with an unchanged network in which the decentralisation level (DL) is 0 . With every increase of $D L$ by 1 , the aggregated generator capacity at a single node in the original network will be replaced by three identical DGs, until DL reaches 9. Thus, with the replacement of an original generator, the total generation capacity of a node will be distributed over three newly added nodes representing DGs. After each generator replacement, the generator impact and capacity imbalance will be updated. At a given $\mathrm{DL}$, one simulation is carried out by running the power flow model of the corresponding network over a whole year. The time step, which determines the number of completed power flow runs within one simulation, varies in this study and will be stated in each section separately. A simulation set is defined as a complete set of simulation results in which nine generators have been replaced by DGs. As every simulation involves random factors, 30 repeated simulation sets are run for each strategy to generate statistics for comparison.

\subsubsection{Part II: Systems with intermittent DGs and a dynamic demand profile}

For the DGs based on renewables such as wind and solar energy, the intermittency of the energy resource has to be considered. As for Part I, a random strategy and strategies 1 to 3 for introducing DGs are adopted, and each strategy is studied with 9 DLs and 30 repeated simulation sets. However, the intermittent nature of the DGs and the consideration of a dynamic demand profile require several additional treatments, as explained below.

\section{Capacity credit factor for replaced generators}

Because of the intermittency, simply replacing the original generation capacity by DGs - as in Part I will certainly lead to power imbalances. Therefore, a capacity credit factor (CCF) is introduced for an intermittent generator, reflecting its firm capacity equivalence. The original generator capacity, kept at its original location in the network, will not be replaced completely, but only to the extent of the CCF of the DG. Thus, the retained portion of the original generator represents a sort of reserve capacity in the grid. The CCF is a value in the range of 0 to 1 . If CCF equals zero, it is assumed that the DG offers no credit for reducing the original capacity of the generators. In other words, $100 \%$ of the original capacity of the replaced generator will be required to be kept online. In contrast, a CCF of 1 means that the DG offers the potential to replace the full capacity of the original generator, or in other words, $0 \%$ of the original capacity of the replaced generator will be kept online. 


\section{Energy storage}

Another way to balance supply and demand is to incorporate energy storage in the electricity grid. Since energy storage can offer a broad range of functions, it highly depends on the actual technical, economic and regulatory issues to select the optimal size and locating of energy storage devices [37]. Some simulation results show that "co-locating wind and storage enhances the performance of wind integration. However, the optimal solutions for reliability enhancement with higher wind penetrations require placing the storage at a bus other than the wind bus" [38]. Another study, focusing on transmission costs of wind energy combined with compressed air energy storage, concludes that there "appears to be significant number of cases of transmission costs that warrant co- locating wind with storage. However, co-location of wind and storage will be less attractive if a load-sited storage device is able to take advantage of high-value ancillary or capacity services" [39]. Focusing on the integration of wind energy with hydrogen systems, offers again other technical and economic considerations [40]. Ref [41] finds that the optimal storage placement depends mainly on the line-flow limits and therefore on the network structure and is robust to the position of renewable generation in the network and to the ratio of conventional to wind generation. Moreover, the amount of energy storage needed when the number of variable renewable sources increases in a grid, is a complex issue. In reality, this is an economic issue and depends on the "integration costs of variable generation and the amount of various storage or other enabling technologies that are economically viable in a future with high penetrations of variable generation" [42]. The sometimes-conflicting conclusions confirm the "lack of coherence in the modeling approaches used and recommendations drawn by the community. These discrepancies arise in large part due to the versatility of storage resources and the market and regulatory structures that determine storage economics" [37].

Energy storage is included through the following simplified treatments:

- For each DG, an extra node representing decentralised energy storage is added to the same node where the DG has been connected to. Any surplus in the power supply of DGs will be stored in the corresponding energy storage node. Locating a battery near a DG is already common practice, since it allows the DG owner to have more flexibility in selling the energy at most cost-effective moments. Also, it gives a network operator more control over the power quality. Moreover, storing energy at a different node in the network would require to model power rerouting for this surplus power, which will further complicate this study. Therefore, unlimited storage capacity has been taken as a simplification, which is considered as being in line with the assumption that network operators will create incentives which ensures that the system operates at the lowest cost to help avoid problems and the need for too much spare capacity.

- If stored energy is available for a specific energy storage node, the maximum supply capacity is set to the maximum generation capacity of the corresponding DG, so that energy storage only complements the available local capacity of the related DG, since we are focusing on the impact of the locations of DGs. Moreover, the power lines of the energy storage are sized to be identical to the lines of the corresponding DG. 


\section{Energy demand and renewables supply data}

In order to simulate fluctuations in electricity demand, the hourly demand data from the UK over the year 2015 will be applied ${ }^{2}$. Renewable power production profiles from wind turbines and from solar PV have been modelled based on hourly weather data from a meteorological station in Vlissingen in The Netherlands over the year $2015^{3}$, as shown in Figure 2. The data have been scaled in such a way that wind and PV produce the same amount of energy over a year according to Eq. 1 with $C_{g}=1$. With a focus on a Western European situation and an intention to study at a more abstract level, we are more interested in a representative weather profile to exemplify the intermittency of renewables, rather than that of a specific country.

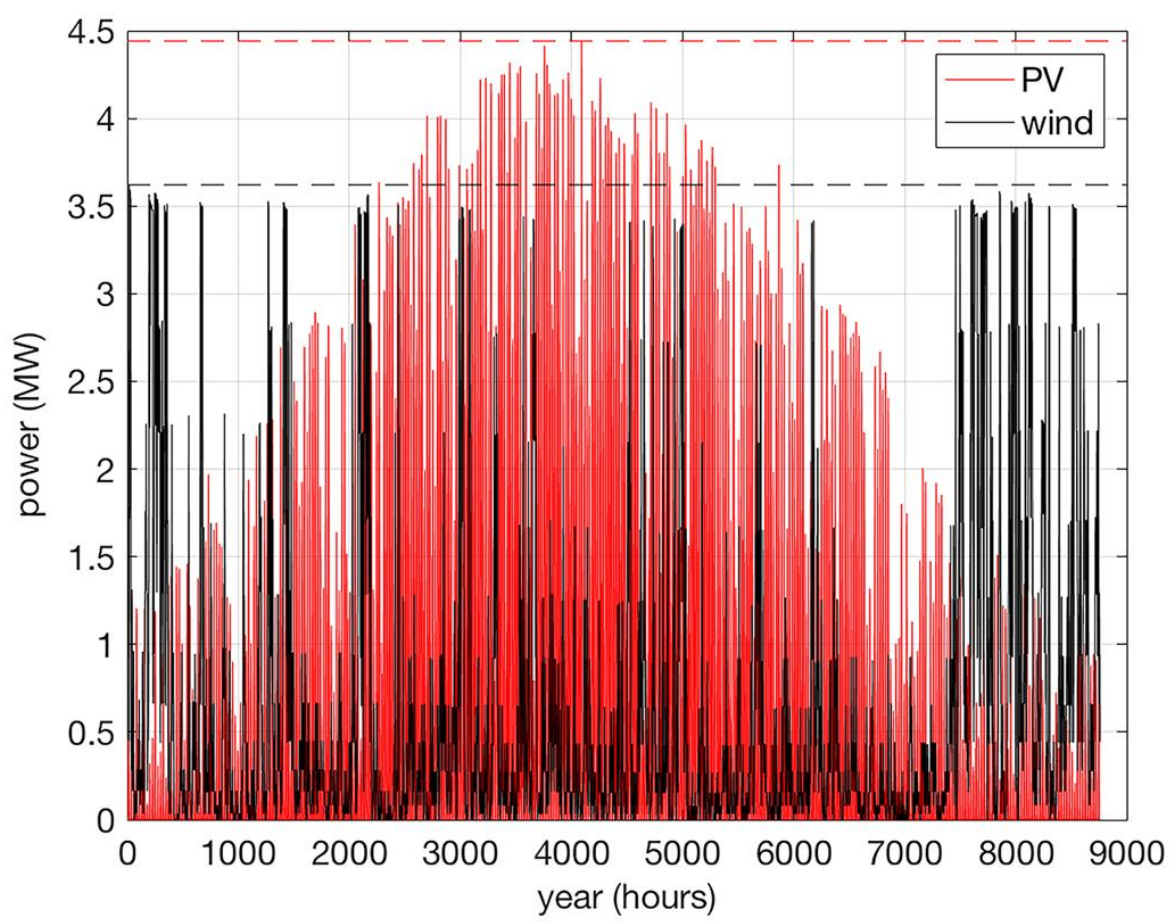

Figure 2: Power profile of hourly PV and wind power scaled in such a way that both produce the same energy output over a year, based on weather data from Vlissingen, The Netherlands 2015. Source: The Royal Netherlands Meteorological Institute (KNMI).

\section{Sizing and connecting DGs}

Similar to the case with the conventional DGs, the total demand in the grid is scaled in such a way that the peak load during the year matches the total generation capacity by applying a demand factor, $f_{d}$. The model deliberately sets the capacity margin to zero in order to stress the system so that some blackouts will occur in the unchanged network. Without this it will be difficult to assess whether decentralisation has potential to improve the network reliability.

Each DG to introduce is alternately a wind turbine or a solar energy plant, starting with a wind turbine. When the DGs would replace an original generator based on the size of capacity, so that the

\footnotetext{
${ }^{2}$ http://www2.nationalgrid.com/UK/Industry-information/Electricity-transmission-operational-data/Dataexplorer/

${ }^{3}$ http://projects.knmi.nl/klimatologie/daggegevens/selectie.cgi
} 
combined nominal capacity of the DGs is the same as the replaced capacity, it is obvious that this would lead to power shortages when the output of DGs is low (e.g. due to low wind speeds) while power demand is high. Hence, in this study, the nominal capacity of DGs will be based on the actual energy output over a year matched with the actual energy output of the replaced generator. As an alternative, one could size the DGs such that it equals the maximum possible energy output of the replaced generator (i.e. running at full capacity all time), however, this would lead to frequent large power surpluses and thus availability of abundant stored energy. With abundant stored energy, the whole network acts more or less like incorporating DGs with a steady capacity, which would not differentiate from the case investigated in part I. To avoid these issues, we will use the actual energy output as a measure to size the DGs. To be precise, the nominal electricity from the DG $k$ to be supplied at hour $i$, denoted as $N_{k, i}$, is sized in such a way that the annual energy output of the DG matches the part of the total network annual demand that is originally to be met by the replaced conventional generator $g$ according to the ratio of the replaced generation capacity, $C_{g}(\mathrm{~W})$ to the total capacity of the conventional generators in the original network, TC (W):

$$
N_{k, i}=C_{g} \frac{P_{R E, i}}{T P_{R E}} \frac{T D}{T C}
$$

where $P_{R E, i}(\mathrm{~W})$ is the renewable power at hour $i$ and $T P_{R E}(\mathrm{Wh})$ is the total annual renewable energy supply, both based on the weather data, and TD is the total annual demand of the network (Wh). For simplicity, the wind and PV data are based on a single location for one specific year, as previously described. To introduce a further degree of randomness in the output by the DGs, the actual hourly output of DG $k$ at hour $\mathrm{i}\left(P_{k, i}\right)$ is determined by applying a random factor $\zeta$ to the nominal value $N_{k, i}$ :

$$
P_{k, i}=N_{k, i} \times \zeta
$$

where $\zeta$ is between $0.8-1.2$.

The DGs are added to the original network as in part I; however, the line capacity of the added lines will be increased to allow an unrestricted flow of power through the line. Since the maximum power generation of renewables is higher than the maximum generation capacity of the replaced conventional generator, as can be seen from Figure 2, the capacity of the added power lines for the DGs will be increased by a line capacity factor (LCF) depending on the type of generator according:

$$
L C F=\frac{\max \left(P_{R E, i}\right)}{T P_{R E}} \frac{T D}{T C}
$$

From the dashed lines in Figure 2 it can be seen that for PV this LCF is roughly 4.5 and for wind this factor is roughly 3.7. The line capacity will be multiplied by the same factors for PV and wind respectively; in addition, it will be multiplied by a factor 1.2 to guarantee that the line rate is large enough for the maximum power flow including the random factor. In this way, constraints due to line flows occur in the original network only, although every line has a small possibility to get tripped by the unwanted operation of relay protection as explained in section 2.1 . 


\section{Active power control}

In reality, PV systems can be disconnected (open circuited); for a wind turbine, this is not a safe option, because it can potentially damage the turbine. Until recently, wind turbines did not have active power control systems; however, due to the recent higher penetration levels of wind turbines, several transmission systems operators require new wind turbines to have a number of active power control capabilities [43]. This study assumes that the dispatching centre also manages the active power control for the DGs based on renewables by diverting surplus power towards energy storage.

\section{Stressing the network}

Preliminary tests in this work showed that blackouts would occur very rarely in the reduced GB network; however, this is mainly related to the power demand profile applied in these simulations: there are just very few moments in the year at which the demand is close to its peak demand. In contrast, in part I the power demand has been assumed to be close to its peak demand all the time.

To be able to simulate the network close to its critical point, there are various failure modes to increase system stress such as failure of generators and failure of transmission lines [25]. Since we focus on line outages in this study, Part II will force one random line outage at the start of each day. The stressed network could lead to other line outages during the day. After each day (i.e. 24 hours), the network gets restored so that each power line is available again.

\section{Merit order}

Although economic costs represent an important aspect during the decision-making process of investments in large scale DGs employment, this study does not focus on such costs per se.

However, we will make use of the generation costs in order to create a certain merit order. The objective function of the optimal power flow model is to minimise the cost of generation while operating within the network constraints. Under specific circumstances the network constraints do not allow to meet all the power demand and some of the loads may become unserved ${ }^{4}$.

In an ideal situation, the conventional generators in combination with DGs plus storage should be able to meet the demand. The use of the replaced generators or reserve capacity should therefore be minimised, but it is still preferred over load shedding. To achieve the preferred merit order, we differentiate the generation cost of each generator type. The ranking, from low to high, is: (1) DGs, (2) original generators, (3) storage, (4) replaced generators and (5) load shed. The replaced generators refer to reserve capacity still available following the introduction of substituting DGs, which can be used as the last resort before load shedding. We have chosen to prefer the use of the conventional generators over energy storage, to increase the likelihood that the first three types of energy generators will be able to meet the demand. If the stored energy would be used before the original generators, it is possible that energy storage had already been depleted and hence would be unavailable during moments of low supply by the renewables. Reserving the stored energy (which, unlike the conventional generation capacity, is not constantly available) as the last resort can be seen as best ensuring a reliable electricity supply from the perspective of a network operator. However, there are also arguments in favour of putting energy storage at the second position. This would give priority to the usage of the energy generated by renewables and thus lowering the overall greenhouse gas emissions. Moreover, when energy storage is owned by private companies or

\footnotetext{
${ }^{4}$ To allow load shedding, MATPOWER internally converts loads to dispatchable generators with a negative power output and a high generation cost, so that it is favourable to keep the 'loads' on whenever possible.
} 
households, then they would prefer to use their stored energy first, before buying electricity from the grid. Nevertheless, if there are benefits for the network operator to use stored energy during delayed moments due to reliability considerations, which are of the focus of this work, they could create incentives to create the merit order as proposed here. Note that due to network constraints (e.g. maximum line capacity), the optimal power flow model could still favour a costlier option over the others to obtain a feasible solution.

\subsection{Case studies}

Since the network structure can have a large impact on the actual network behaviour, two different networks will be applied in this study, namely a representation of the HV transmission grid in Great Britain and the IEEE 39 bus system.

The complete Great Britain transmission network would be cumbersome for study of the effects of increased decentralised power generation, therefore a well-known representative model of the electricity transmission network, the Reduced GB Network as developed at the University of Strathclyde in 2010 [44], is used instead. The rationale behind such a reduced network can be found in [45]. The reduced network consists of 29 nodes $^{5}$, interconnected through 98 transmission lines in double circuit configuration and one single circuit transmission line. Besides, the network consists of 53 generators, so there are possibly multiple generators per node.

To be able to implement the DC power flow model (cf. Section 2.2), the double circuit is replaced by a single circuit configuration. The line capacities are added up, the equivalent line reactance $X_{e q}$ is determined as follows:

$$
X_{e q}=\frac{X_{1} X_{2}}{X_{1}+X_{2}}
$$

The generators connected to one node are aggregated to form a single generator.

In a network, the net power output of each node can be positive, negative, or zero, corresponding to a generation, load or connection node, respectively. Note that a node with a generator and a load might still have a negative net power output when the load is larger than the output of the generator. The two network topologies applied in this study are shown in Figure 3 . The diamonds represent nodes with a generator, red coloured nodes have a load and grey nodes have neither a generator nor a load.

\footnotetext{
${ }^{5}$ In power networks, busbar or bus is a metallic strip or bar for local high current power distribution. It links various power lines with each other to form a power network. In network theory, a more general term 'node' is used, which refers to a point in the network where multiple lines (i.e. edges) connect with each other. In this study, we use both terms interchangeably.
} 


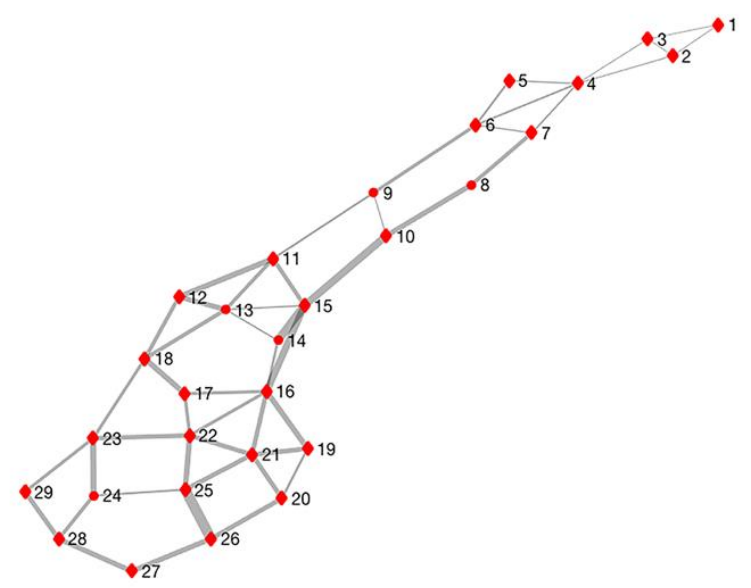

Reduced GB network

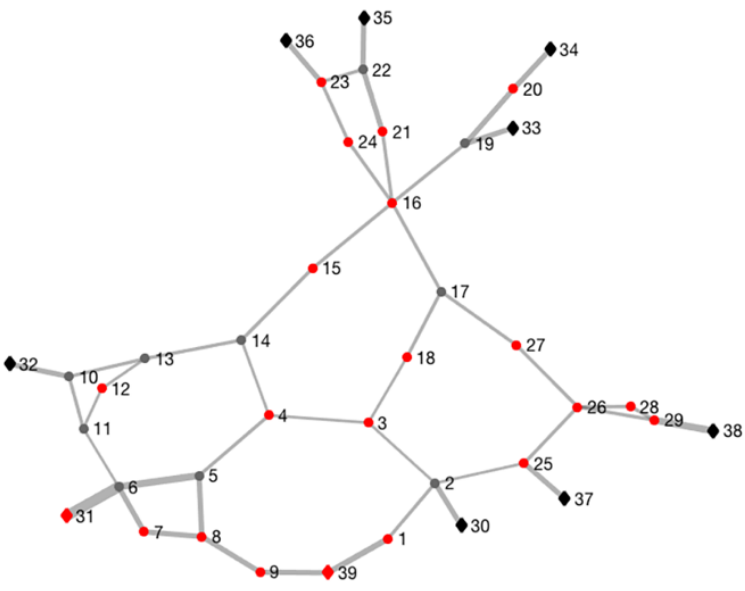

IEEE 39 network

Figure 3: The network structures of the reduced GB network (left) and the IEEE 39 network (right). The line thickness is a measure for the line capacity.

In the reduced GB network ${ }^{6}$, the total load is set to be equal to the total generation capacity in order to simulate a system that operates close to its critical point of operation. For the IEEE network, it has been found by trial and error that this corresponds to $93 \%$ of the total generation capacity. Above this value, a small blackout size will be found which is not related to the decentralisation and therefore has been avoided in this analysis.

InTable 3 an overview is given of the main properties of the networks, based on indices from network theory [46]. The diameter of a network is the greatest distance between any pair of nodes in a network, based on the shortest path between the nodes. The degree of a node is the number of lines connected to it. The density of a network is the fraction of potential lines actually present. The efficiency $(E)$ of a network is a measure of how efficiently a network exchanges information and is defined as [47]:

$$
E(\boldsymbol{G})=\frac{1}{N(N-1)} \sum_{i \neq j \in \boldsymbol{G}} \frac{1}{d_{i j}}
$$

where $N$ is the number of nodes in the network $G ; d$ is the shortest distance from node $i$ to node $j$. The betweenness is defined as the number of shortest paths from all nodes to all others that pass through that node.

Table 3: Overview of basic network properties of the two evaluated networks

\begin{tabular}{|l|r|r|}
\hline \multicolumn{1}{|c|}{ Network } & \multicolumn{1}{c|}{ IEEE 39 } & \multicolumn{2}{c|}{$\begin{array}{c}\text { Reduced GB } \\
\text { converted }\end{array}$} \\
\hline Number of nodes & 39 & 29 \\
\hline Number of generators & 10 & 24 \\
\hline Number of loads & 21 & 29 \\
\hline Number of lines & 46 & 50 \\
\hline Total maximum generation capacity (kW/MW) & $7,367.0$ & $82,384.8$ \\
\hline
\end{tabular}

\footnotetext{
${ }^{6}$ The orientation of the plotted reduced GB network does not correspond exactly to the actual geographical locations: the small top part of the network represents the northern part of Great Britain (i.e. Scotland), while node 25 represents London.
} 


\begin{tabular}{|l|r|r|}
\hline Total load (kW/MW) & $6,254.2$ & $56,325.9$ \\
\hline Diameter & 10 & 11 \\
\hline Average degree & 2.4 & 3.4 \\
\hline Minimum degree & 1 & 2 \\
\hline Maximum degree & 5 & 6 \\
\hline Density & 0.0310 & 0.0616 \\
\hline Efficiency & 4.75 & 3.95 \\
\hline Average betweenness & 71.2 & 4.1 \\
\hline Minimum betweenness & 0 & 0 \\
\hline Maximum betweenness & 334.3 & 123.3 \\
\hline
\end{tabular}

As can be seen from Figure 3 and Table 3, the IEEE 39 network is less dense than the reduced GB network. Besides, each generator has a degree of 1 except for the generator at bus 39, which makes the network more decentralised than the reduced GB network. Generators at the busses 31 and 39 have a load as well. Furthermore, there are 21 loads in total, which means that 10 nodes are neither a generator nor a load. So, this network is in many aspects different from the reduced GB network, whereas the latter is a more aggregated version of a real network.

The average betweenness is larger for the IEEE network, indicating that the network includes specific nodes which play a prominent role in distributing power flows. A lower average betweenness, as shown for the GB network, indicates that the network flows will be distributed more evenly. However, in reality, power does not have to flow via the shortest paths, therefore simulations of the power flow are required to study the actual network behaviour. 


\subsection{Overview of the procedure for adding DGs}

Figure 4 presents an overview of the network configuration to be simulated in each part of the study; the figure shows only the GB network but the same is done for the IEEE 39 network.

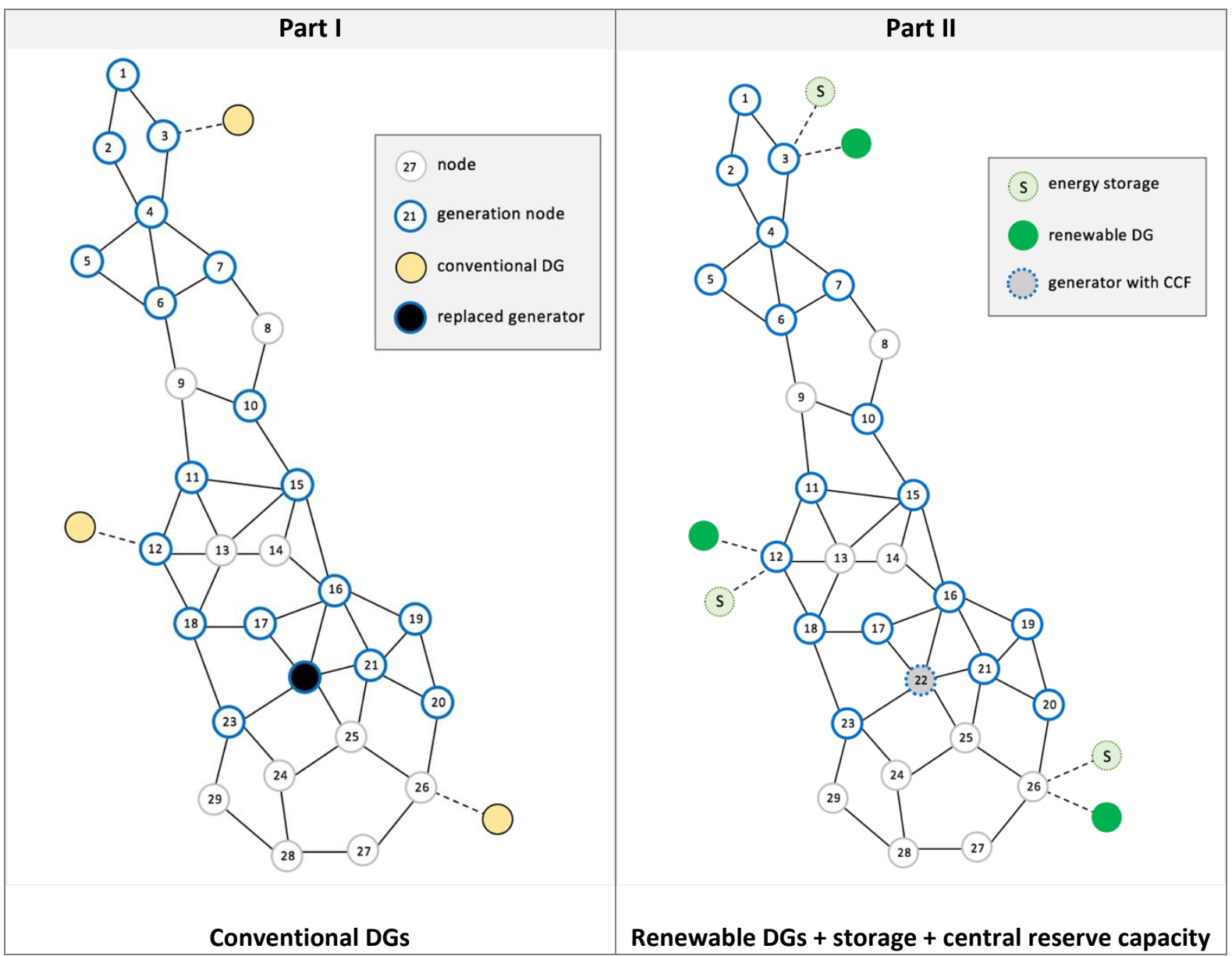

Figure 4: Overview of simulated network configurations, showing the first decentralisation level with 3 DGs added to the network, including storage and reserve capacity for part II.

The detailed procedures for adding new nodes to the original network for both the DGs based on conventional and for DGs based renewables are shown in Table 4. 


\begin{tabular}{|c|c|c|}
\hline Related to & Introducing conventional DGs (Part I) & Introducing renewable DGs (Part II) \\
\hline $\begin{array}{l}\text { Selected } \\
\text { original } \\
\text { generator }\end{array}$ & - $\quad$ Set the generator capacity to zero & $\begin{array}{l}\text { Multiply the generator capacity by } \\
\text { (1-CCF) and set the generation cost } \\
\text { to } G C_{\text {replaced }}\end{array}$ \\
\hline \multirow{2}{*}{$\begin{array}{l}\text { The three } \\
\text { newly added } \\
\text { DGs }\end{array}$} & \multicolumn{2}{|c|}{$\begin{array}{l}\text { - } \quad \text { Add a new generation node } \\
\text { - } \quad \text { Set the capacity of the DG equal to a third of the original generator capacity } \\
\text { - } \quad \text { Connect the DG to the selected original node through a new power line } \\
\text { network }\end{array}$} \\
\hline & $\begin{array}{l}\text { - Set the line rating to } 1.2 \text { times the } \\
\text { DG's capacity }\end{array}$ & $\begin{array}{l}\text { - Set the line rating to } 1.2 \text { times the } \\
\text { DG's capacity times the PV or wind } \\
\text { factor (i.e. } 4.5 \text { or } 3.7 \text {, respectively). } \\
\text { - } \quad \text { Set the generation cost to zero }\end{array}$ \\
\hline Storage & & 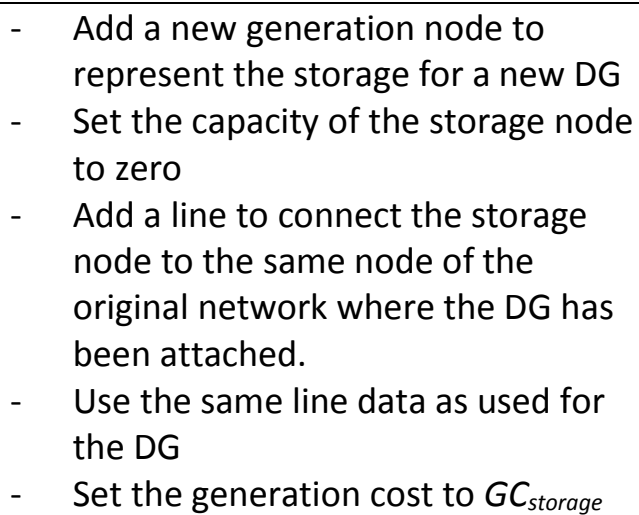 \\
\hline
\end{tabular}

\section{Results}

Earlier in the paper, three different strategies for locating DGs were introduced (Table 2). In this section, only the results contrasting the random approach with strategy 3 are presented in detail to interpret the outcomes with sufficient clarity; further details of the results of the other two strategies can be found in appendix $A$.

\subsection{Part I: conventional DGs}

As mentioned before, Part I treats the DGs as conventional power generators which have a fixed maximum generation capacity. The time step in this section is a day, so each simulation consists of 365 power flow runs. In total 30 simulation sets have been simulated.

In Figure 5 the results of 30 simulation sets for the reduced GB network (left) and the IEEE 39 network (right) are shown in boxplots ${ }^{7}$ for each decentralisation level (DL), for the random approach and strategy 3. To be able to show differences in the results a logarithmic scale has been applied. The median values are shown with a circle with a black dot in the centre; outliers are shown as plain circles. The top two plots show the blackout size relative to the total demand and the two plots at the bottom show the blackout frequency relative to the number of simulations (i.e. 365 ). The grey

\footnotetext{
${ }^{7}$ The boxplot draws points as outliers if they are greater than $q 3+1.5 \times(q 3-q 1)$ or less than $q 1-1.5 \times(q 3-$ q1), where q1 and q3 are the 25 th and 75 th percentiles of the sample data, respectively. This corresponds to approximately $+/-2.7 \sigma$ and 99.3 percent coverage if the data are normally distributed.
} 
area shows the range of median values for the unchanged network. For some of the strategies the blackouts occur very rarely during some of the DLs, therefore the plots show no median values, but only some outliers or even no results at all. For example, in Figure 5a, no results are shown for strategy 3 for DLs $3-6$. The whisker bars are mainly shown for the random strategy in which a larger range of results are obtained.

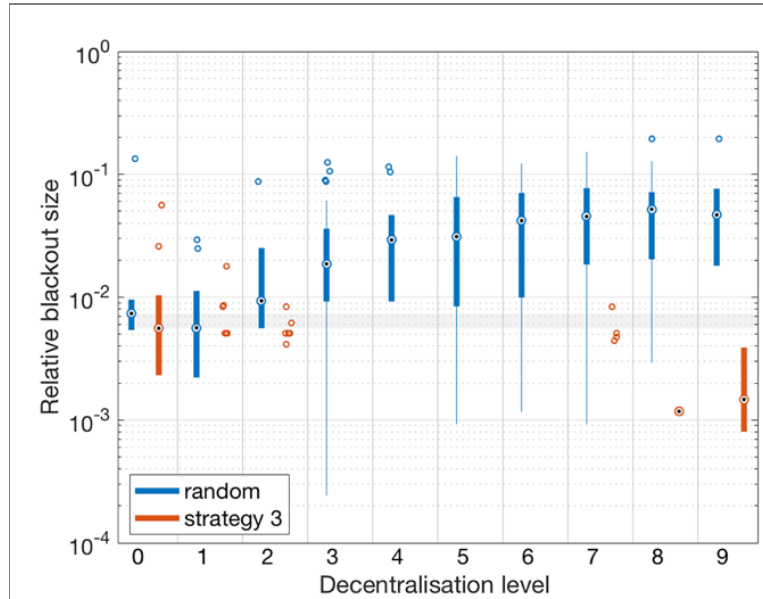

(a)

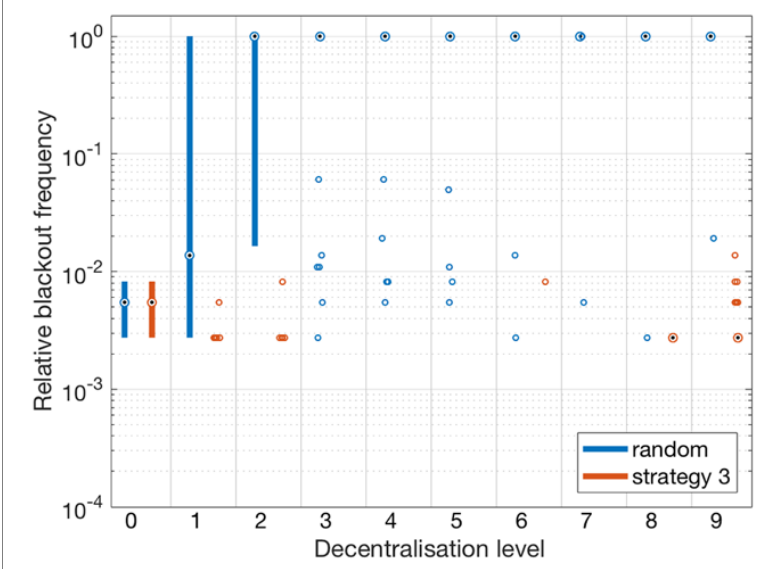

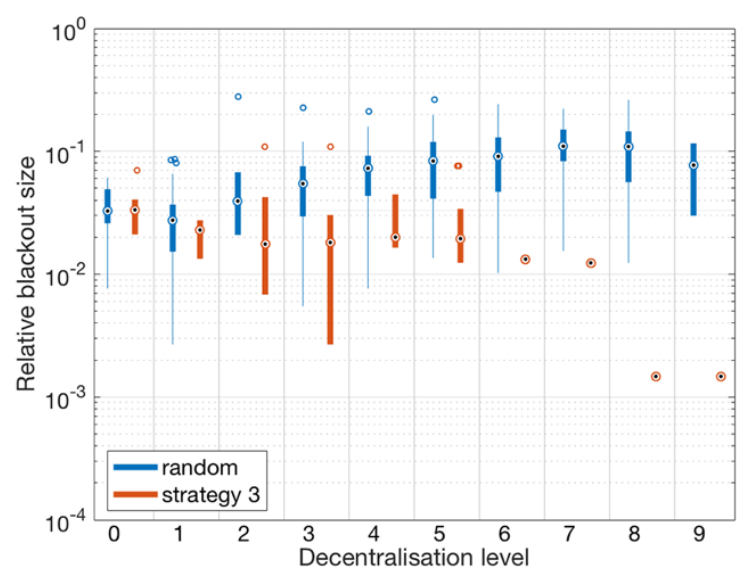

(b)

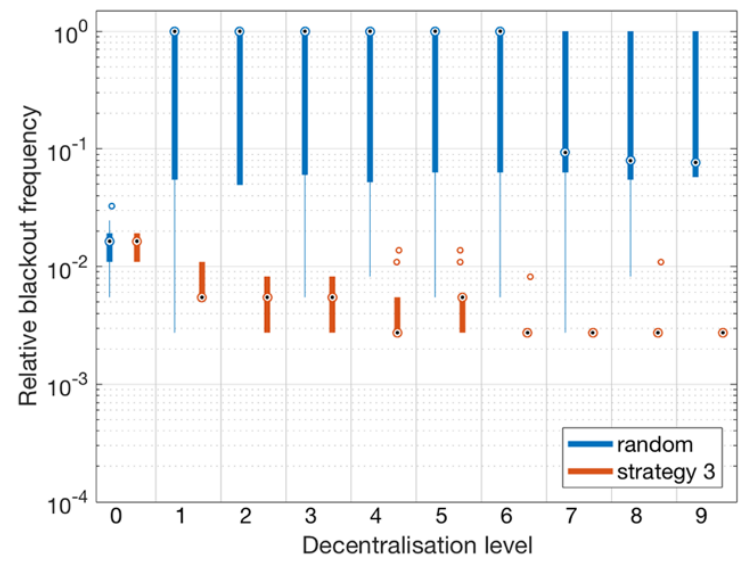

(d)

Figure 5: Relative blackout size (a, b) and relative blackout frequency (c, d) for the reduced GB network (left) and the IEEE 39 network (right) with conventional DGs, based on 30 simulation sets, each simulating a year with a time step of a day, for different levels of decentralisation.

From the figure, it can be seen that the two networks experience different blackout behaviour. First, notice the relative blackout size for the unchanged networks at DL 0 , i.e. when no DGs have been added. The GB network has a rather small average relative blackout size with median values in the range of $0.5 \%-1.0 \%$, whereas the median values for the IEEE network is in the range of $3.0 \%-3.5 \%$.

The relative blackout size for the GB network increases with an increasing DL for the random approach; strategy 3 decreases the blackout size compared with the random strategy. Most of the relative blackout sizes of the strategy 3 remains in the lower region of the grey area or below, indicating a lower blackout size than the unchanged network. In Appendix $A$, the results are also shown for strategy 1 and 2 . Briefly, strategy 1 shows already a significant improvement compared with the random approach, suggesting that the degree of power imbalance plays an important role. 
Compared with strategy 2, which selects the generator with the lowest impact, the variance is larger for strategy 1 which selects a random generator to be replaced. This indicates that the generator impact is also an important factor which influences the blackout statistics. In appendix B, the blackout impact and generator impact have been plotted, showing that there is a strong correlation between the two. Strategy 3 , which selects both the existing generator to be replaced and the location of a new DG strategically, gives the best results.

The results for the IEEE network show a similar trend. The random approach shows higher relative blackout sizes than strategy 3 .

The relative blackout frequency is another measure for reliability. From Figure 5 it can be seen that regarding the GB network, the random approach results often in a blackout. Strategy 3 shows clear and similar improvements. In the case of the IEEE network, strategy 3 gives significantly better results than the random approach. The blackout frequency of the random strategy shows that for some of the simulations a blackout will occur for every power flow run. This indicates that the random replacement of generators and the random additions of DGs will lead to overloaded parts in the network which limits the ability to serve all the demand.

The relative blackout impact, i.e. the product of the relative blackout size and the relative blackout frequency, is shown in Figure 6 for both networks. From this figure, it becomes clear that strategy 3 performs the best for both networks. For the GB network, strategy 3 shows only outliers for the DLs $1-7$ and shows low median values for DL 8 and 9. For the IEEE network, strategy 3 decreases with increasing DL. This suggests that the combined considerations of the power imbalance and the generator impact (each considered by strategy 1 and strategy 2, respectively) as in strategy 3 results in the lowest blackout impact compared with the other studied strategies.
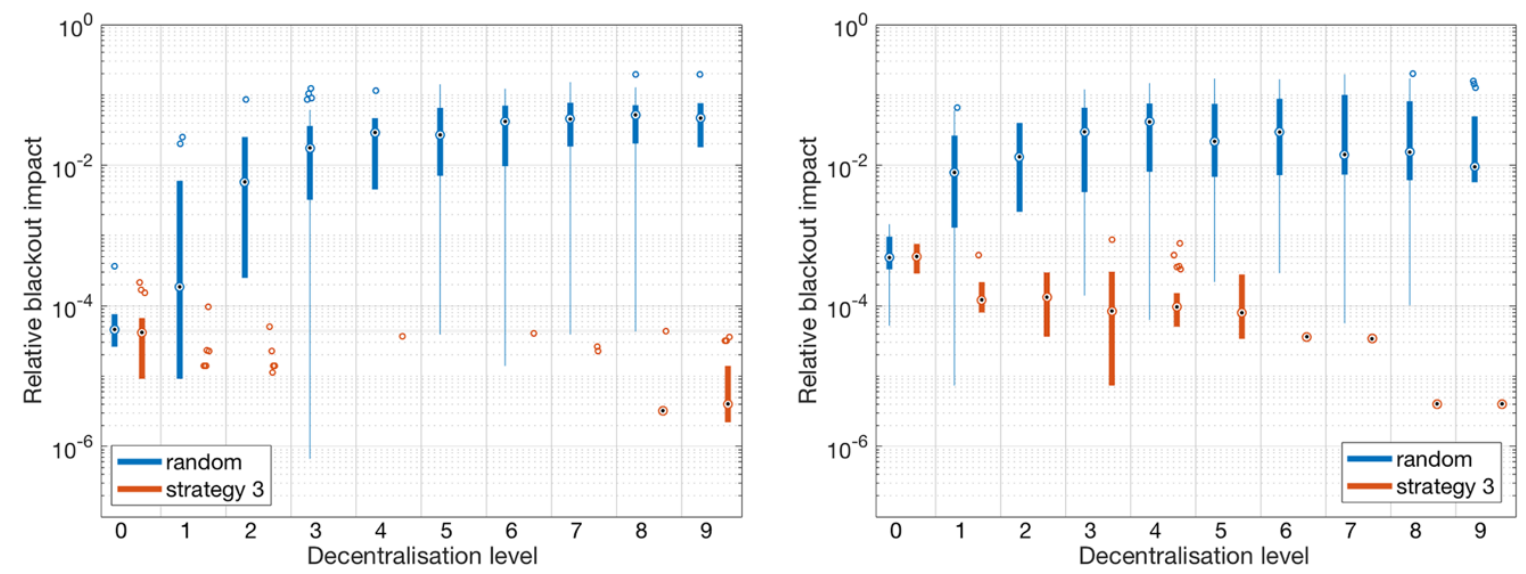

Figure 6: Relative blackout impact for the reduced GB network (left) and the IEEE 39 network (right) with conventional DGs, based on $\mathbf{3 0}$ simulation sets, each simulating a year with a time step of a day, for different levels of decentralisation.

Figure 7 shows the change in the average capacity imbalance relative to the capacity imbalance for the unchanged network.

For the GB network the random approach shows an increase in average capacity imbalance for increasing levels of decentralisation; strategy 3 shows a decreasing trend. 
For the IEEE network, the capacity imbalance remains more or less constant for the random approach and decreases for strategy 3. For DL 1 to DL 4, the capacity imbalance remains more or less constant. After that, the decreasing effect is stronger.
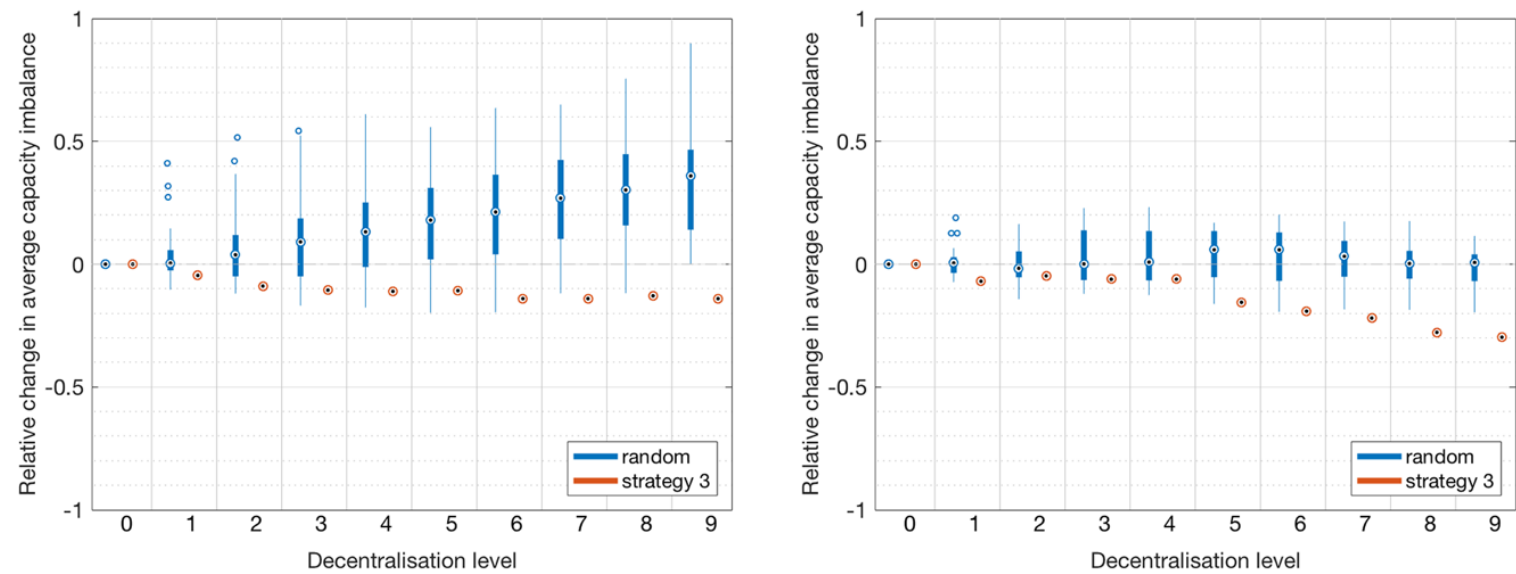

Figure 7: Relative change in average power capacity imbalance for the reduced GB network (left) and the IEEE 39 network (right) with conventional DGs, based on 30 simulation sets, each simulating a year with a time step of a day, for different levels of decentralisation.

Summarising line outages predicted by all the simulation runs in Part I, Figure 8 shows both network structures in which the line thickness of the power lines is a measure for the number of line outages, i.e. the total amount that a specific line has been tripped, during all the blackouts, either randomly or due to line overloading. 


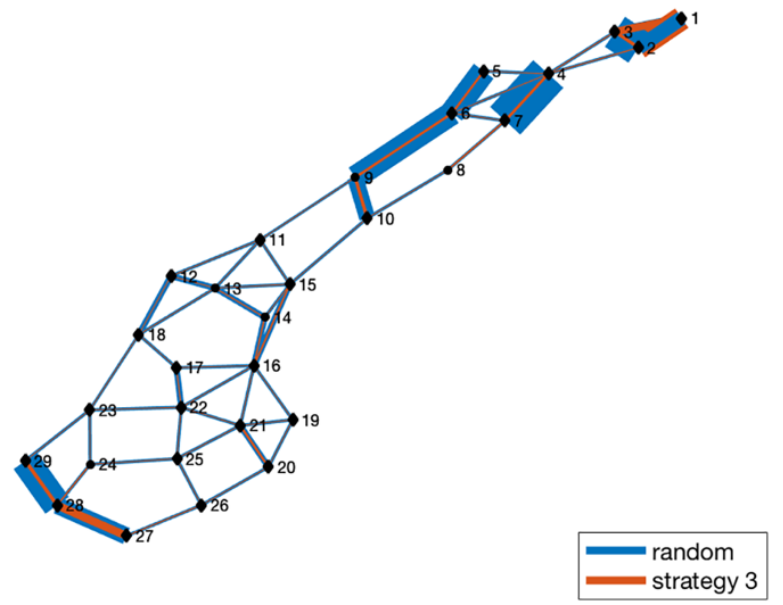

Max: $145(0.13 \%)$

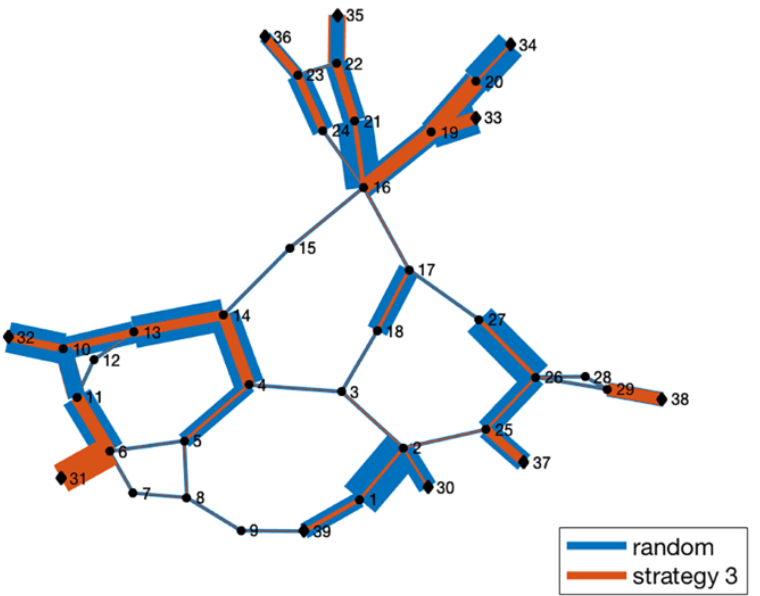

Max: $128(0.12 \%)$

Figure 8: Number of line outages for each line in the network during all the occurring blackouts, for the GB network (left) and the IEEE network (right) with conventional DGs, based on 30 simulation sets, each simulating a year with a time step of a day, for the random approach and strategy 3. In order to present the outcome of both strategies in one figure without losing much information, the strategy resulting in the highest number of outages for the specific power line, represented with a line with the greatest thickness, is plotted first, which is then overlaid sequentially by the symmetrically centred lines with reduced thickness representing the other strategy, in the descending order of the power line outage number. Therefore, the line colour visible at the edges of a line between two nodes corresponds to the first plotted strategy, i.e. the one with the largest number of power line outage. Conversely, the line colour visible at the centre of the line, plotted last, corresponds to the strategy with the lowest amount of line outages. The extra added lines are not included in the figure, since in the applied model the probability of line tripping for these lines is set to be very low. Moreover, excluding the extra lines will increase the readability of the figure. The thickness of each line is relative to the maximum number of line outages of a specific line during a specific strategy per network. The line thicknesses of the GB network are therefore not comparable with those in the IEEE network.

From Figure 8 it can be seen that in the GB network the line connecting the nodes 4 and $7,1_{4-7}$, trips the most for the random approach. Furthermore, $I_{1-2}, I_{1-3}, I_{2-3}, I_{5-6}, I_{6-9}, I_{9-10}, I_{27-28}$ and $I_{28-29}$ show a significant larger frequency of line outages compared to the other lines. Why are some lines more vulnerable than others, as reflected by their instances of line outages recorded during blackouts? An exact explanation is hard to give. However, comparing the results with the line capacities as schematically shown in Figure 8, it is clear that the power lines connecting the nodes 1 to 11 (i.e. the lower region) have a lower capacity than the rest of the network. Moreover, this part of the network is also less well connected. This combination makes this region more vulnerable to blackouts if a line outage happens. The lines connected to node 1 have a very limited capacity, leading to an immediate blackout if one of these lines experiences an outage, since there is just one path left to supply power to node 1 , which also affects $\mathrm{I}_{2-3}$. This rationale does not explain the outages of the power lines $I_{27-28}$ and $I_{28-29}$ directly, but it seems likely that an outage in one of those lines will cause a change in the network flows which will in turn put more pressure on the lower region of the network, eventually leading to a blackout.

The colour order of most lines shows that strategy 3 decreases the outage frequency; however, this does not hold for every line. For example, $I_{1-2}$ and $I_{1-3}$ show the largest frequency for strategy 3.

Compared to the reduced GB network where the outage vulnerability concentrates on a small fraction of the power lines, the IEEE network shows more lines which experience a line outage. The "ring" from node 5 to 6 via node 13 is formed by relatively low capacity lines. Therefore, it is likely that if one of these lines experiences an outage, not all the needed power can be rerouted to meet the demand, leading to a blackout. Furthermore, the majority of generators are connected at the 
endpoints of the network, if for example ${ }_{123-24}$ or $\mathrm{I}_{21-22}$ trips, the generation capacity is not available to the rest of the network which can cause a blackout.

Furthermore, the random approach does not always result in the largest line outage frequency, see for example $I_{4-5}, I_{10-11}, I_{22-35}$ and $I_{25-37}$, however, strategy 3 shows the lowest frequency for most lines.

In general, the results in Part I show that the applied strategy performs better than the random approach and that strategy 3 often also outperforms the unchanged network, indicating that decentralisation improves the reliability of a network.

\subsection{Part II: renewable DGs}

Following the approach presented in Section 2.3.2, simulations are performed on introducing DGs based on intermittent renewable energy resources into the two studied networks while meeting a dynamic demand. Note that, as previously proposed, energy storage will be used in this case, and a random line of the original network will be forced to have an outage at the start of each day, in order to simulate a network operating closer to its critical point.

Energy storage requires simulating a continuous period of electricity demand and supply in order to determine whether a surplus of electricity will be generated, therefore the approach used in Part I cannot be applied here. In this Part II we will use data of a full year instead with a time step of one hour. Results are presented first for the simulations where the capacity credit factor (CCF) of each DG is set to zero and hence a "replaced" original generator is still kept online with its full original capacity. The studies on the impact of varying CCF will be presented subsequently ${ }^{8}$.

\subsubsection{Results with CCF set to zero}

Figure 9 shows the results for the random approach and strategy 3 for the GB network and the IEEE network. The random approach applied to the GB network shows an increasing blackout impact for increasing levels of decentralisation; the blackout impacts for strategy 3 show a slight increase for increasing DLs. Both the random approach as strategy 3 show an increase in blackout impact compared with the unchanged network.

\footnotetext{
${ }^{8}$ Some optimal power flow runs could not be solved within the maximum amount of iterations, the number of these unsolved cases are shown in Appendix C.
} 


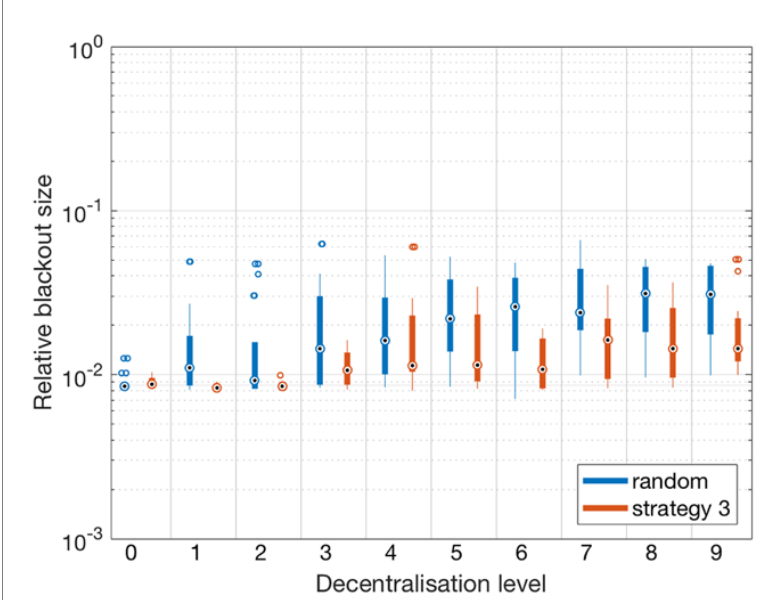

(a)

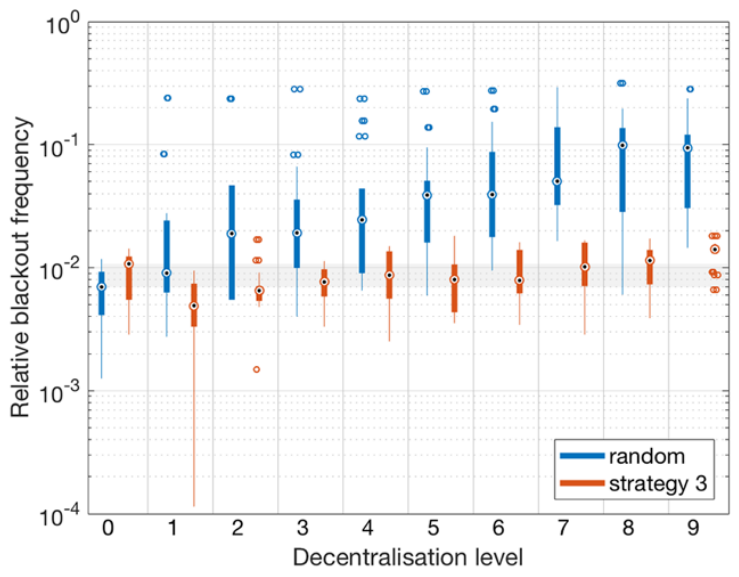

(c)

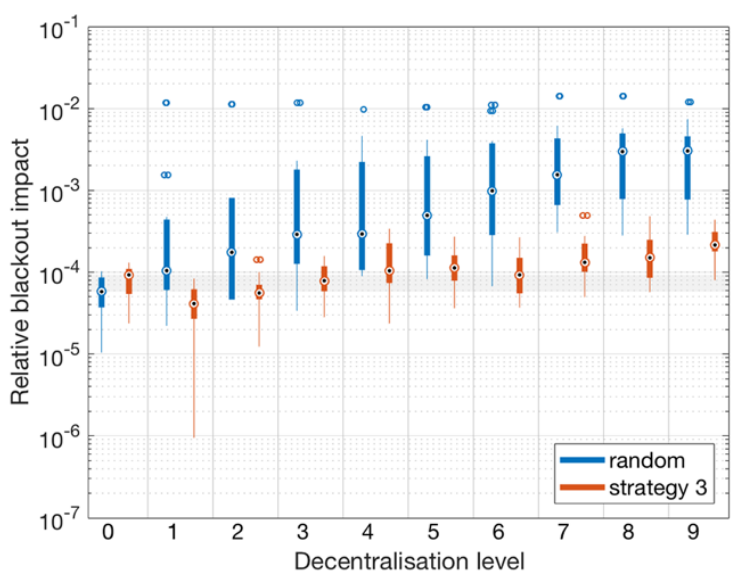

(e)

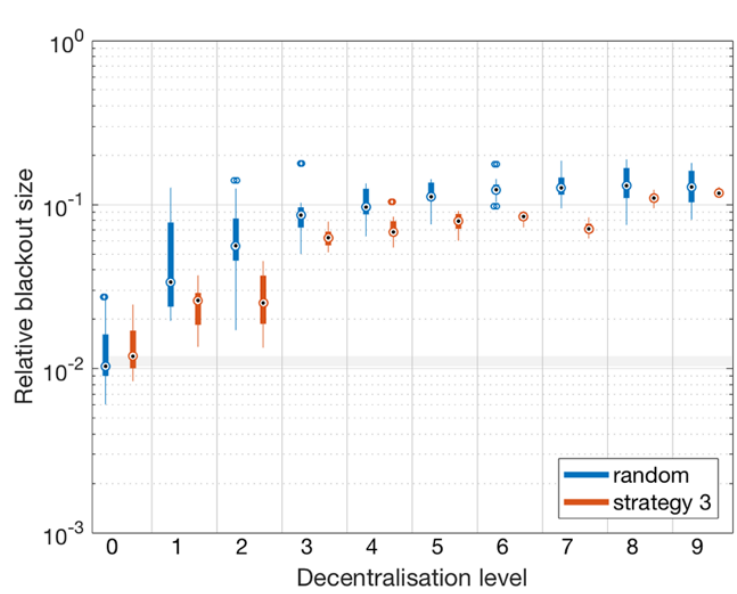

(b)

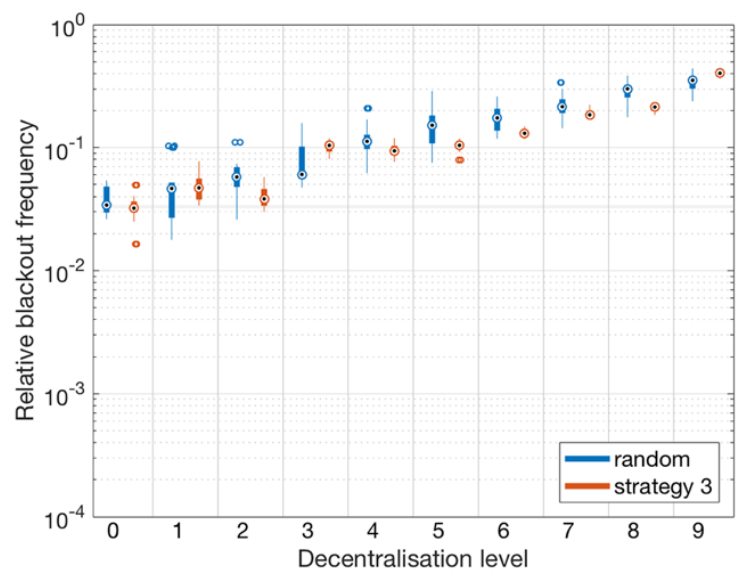

(d)

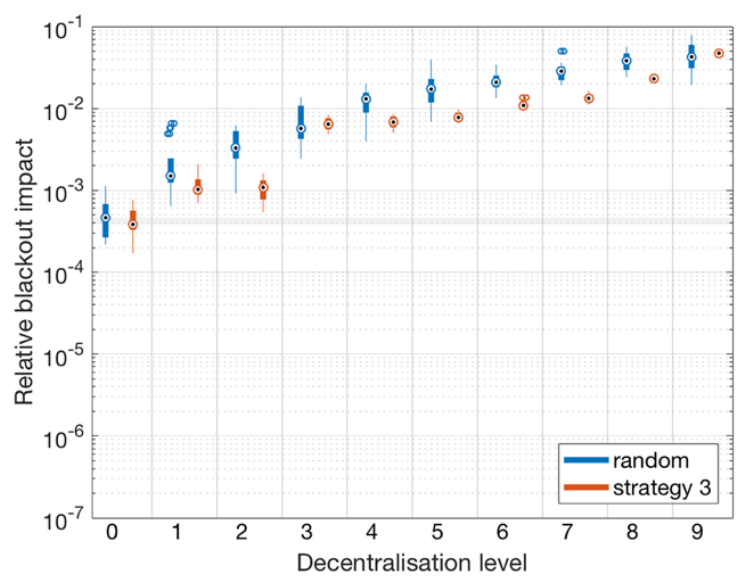

(f)

Figure 9: Relative blackout size (a, b), relative blackout frequency $(c, d)$ and relative blackout impact (e, f) for the reduced GB network (left) and the IEEE 39 network (right) including renewable DGs with decentralised storage, based on 30 simulation sets, each simulating a year with a time step of an hour, for different decentralisation levels for a CCF of $0 \%$.

For the IEEE network, the relative blackout impact also increases with increasing values for DL for each strategy. Strategy 3 gives often the best results, however the differences with the random approach are less distinct. 
The results suggest that networks with decentralised generators based on renewables (including decentralised energy storage, and with original generation retained as backup) are more vulnerable when exposed to initial line outages than the unchanged network. At the first glance, this observation seems to be counterintuitive: since CCF is set to $0 \%$, there is enough supply guaranteed to meet the demand, even if there would be no energy storage available and if all the DGs would be supplying no power at all. A key factor to solve this puzzle seems to be the inclusion of the probability that the dispatch centre does not operate. When the dispatch centre fails, the optimal power flow will not be executed, the power output of DGs is not properly routed, and at peak moments some DGs will cause overloaded lines, leading to one or more lines getting tripped. This in turn makes the network weaker with some nodes becoming isolated, causing further difficulties in balancing supply and demand and thus more load shedding. To validate this rationale, the random approach and strategy 3 have been simulated additionally with the dispatching centre always operating. The results are shown in Figure 10, where the grey area corresponds to the median blackout impact from Figure 9 as comparison.

From Figure 10 it can be seen that the blackout impact regarding the GB network reduces significantly and that strategy 3 performs better than the random approach. Compared with the blackout impact of the unchanged network with the normal model, as indicated as reference by the grey area in Figure 10 (i.e. median values slightly below $10^{-4}$ ), the blackout impacts of strategy 3 with the dispatching centre always operating, remain lower than the unchanged network. Although the random approach shows results above $10^{-4}$ with increasing decentralisation levels, the blackout impacts are significantly lower compared with the values in Figure 9. However, the random approach will result in a slightly less reliable network compared with the unchanged network for high decentralisation levels, even if the dispatching centre functions always.
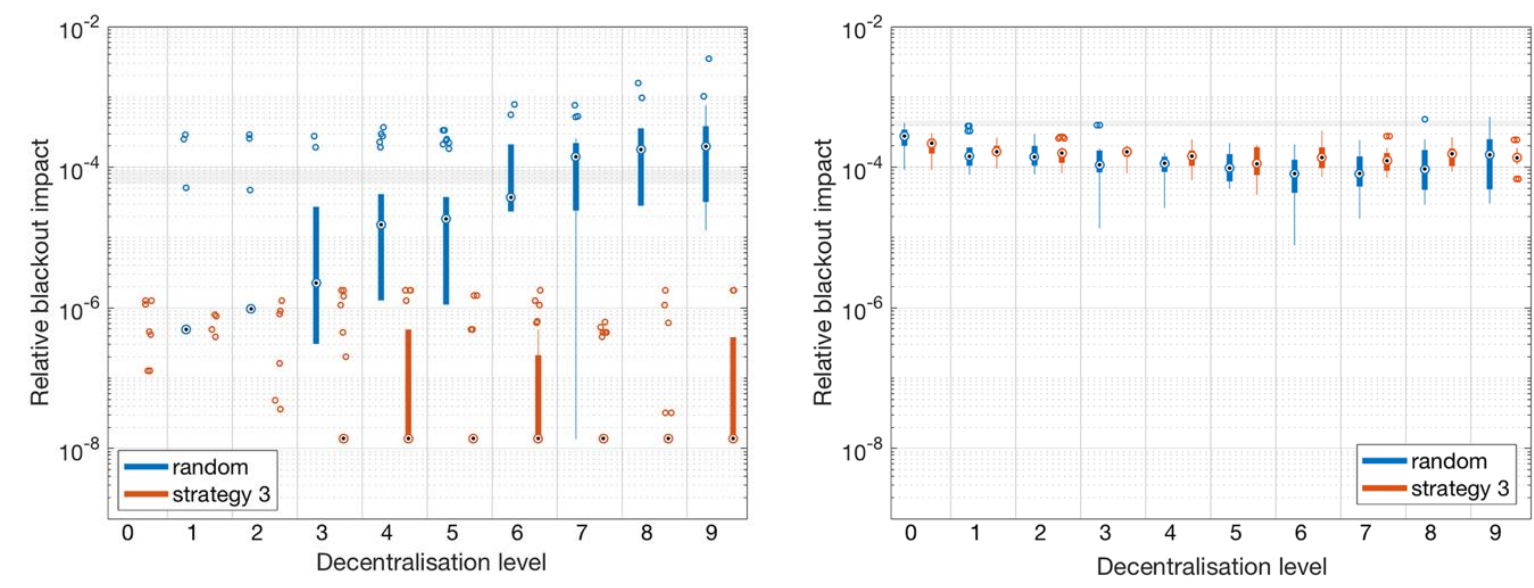

Figure 10: Relative blackout impact for the reduced GB network (left) and the IEEE 39 network (right) including renewable DGs with decentralised storage, with the dispatching centre always operating, based on 30 simulation sets, each simulating a year with a time step of an hour, for different decentralisation levels for a CCF of $0 \%$.

For the IEEE network, the results in Figure 10 also show a decrease compared with the results in Figure 9. It follows that the results for the IEEE network show a slight decrease in blackout impact compared with the unchanged network and thus an increase in reliability. Also, the random approach does not give significant worse results and performs for specific DLs even better than strategy 3. In general, the blackout impacts for the IEEE network are significantly lower compared with the results in Figure 9. 
Overall, comparing the results of Figure 9 with Figure 10 shows that the correct operation of the dispatching centre is crucial for maintaining reliability for both networks as a greater variety of decentralised and intermittent assets are added.
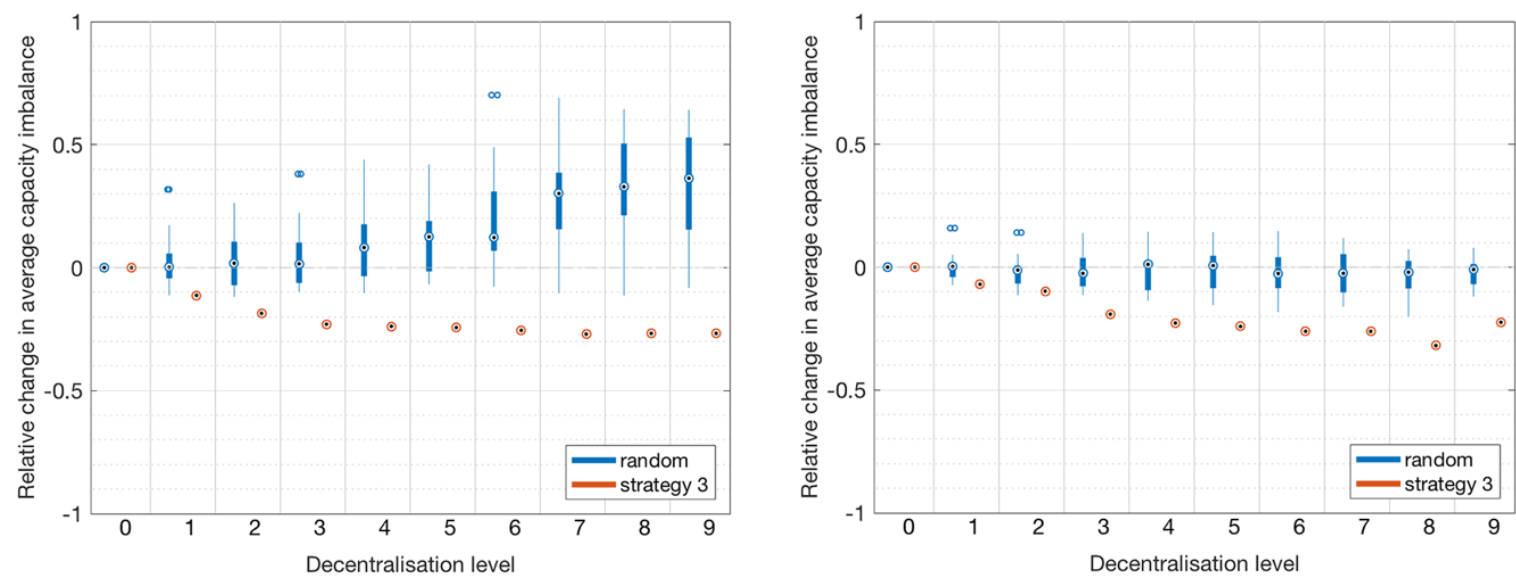

Figure 11: Capacity imbalance for the GB network (left) and the IEEE network (right) including renewable DGs with decentralised storage, based on 30 simulation sets, each simulating a year with a time step of an hour, for different decentralisation levels for a CCF of $0 \%$.

Figure 11 shows the relative change in average capacity imbalance for both networks. For the GB network, the random approach increases the imbalance. Strategy 3 decreases the imbalance with increasing DL. The results are comparable with the results for the conventional DGs.

From Figure 12 it can be seen that $\mathrm{I}_{1-2}$ and $\mathrm{I}_{1-3}$ are most vulnerable in the GB network. The $\mathrm{I}_{1-3}$ has a line capacity of $264 \mathrm{MW}$, which is relatively low compared with the rest of the system. Once $\mathrm{I}_{1-2}$ would experience an outage, all the power flow to or from node 1 should flow via $I_{1-3}$, which will cause a blackout (i.e. load shed).

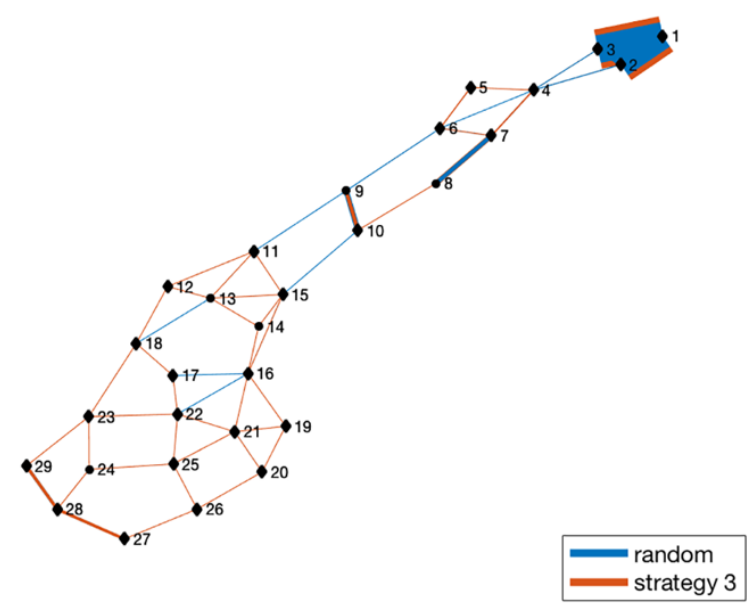

Max: $24,729(0.9 \%)$

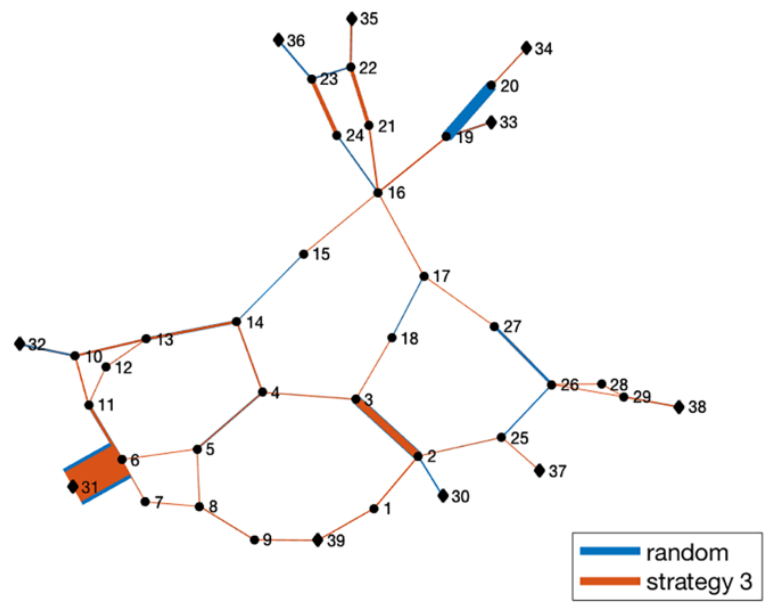

Max: $64,809(2.5 \%)$

Figure 12: Number of line outages for each line in the network during all the occurring blackouts, for the GB network (left) and the IEEE network (right) including renewable DGs with decentralised storage, for different decentralisation levels and different strategies for a CCF of $0 \%$, based on $\mathbf{3 0}$ simulation sets, each simulating a year with a time step of an hour.

For the IEEE network, $I_{6-31}$ is most frequently tripped. As also noted in Part I, node 31 has also a load, therefore if $\mathrm{I}_{6-31}$ experiences an outage, it will always result in a blackout. 
The results presented in Figure 12 show that the vulnerability concentrates on a fewer lines compared to the results corresponding to conventional DGs as presented in Figure 8 in Part 1. This is especially the case for the IEEE network. Moreover, the differences in the amount of line outages between the random approach and strategy 3 are less clearly visible. This could be explained by the difference in stressing the network. Since the load profile applied in part II fluctuates, the loading levels of the power lines are less critical. However, since a random line is forced to have an outage, mainly lines which cause a direct blackout as a result of this are pronounced in Figure 12. This confirms that the overall loading level of the network is an important factor, which relates to how easy the network can cope with a line outage by redistributing power via other lines.

\subsubsection{Energy storage}

The accumulated total energy storage (in MWh or $\mathrm{kWh}$ ) is the net accumulation of the energy stored over all the decentralised energy storage systems in the network and accumulated since the start of a year. At a specific hour in a year, this total energy storage is determined by the history of surplus energy that enters energy storage and the history of energy withdrawal from storage due to consumption. For an easy comparison between the two studied networks, the amount of total stored energy is shown relative to the maximum hourly load in a network, which is $82,236 \mathrm{MW}$ and $6,805 \mathrm{~kW}$ for the GB network and IEEE network, respectively, and therefore this relative stored energy has the unit of hours ( $h$ ). In other words, the stored energy has been expressed as the number of hours the energy storage can meet the annual peak load. In Figure 13, for each of the 30 simulation sets a curve is drawn to show the relative stored energy profile during a simulated year. 


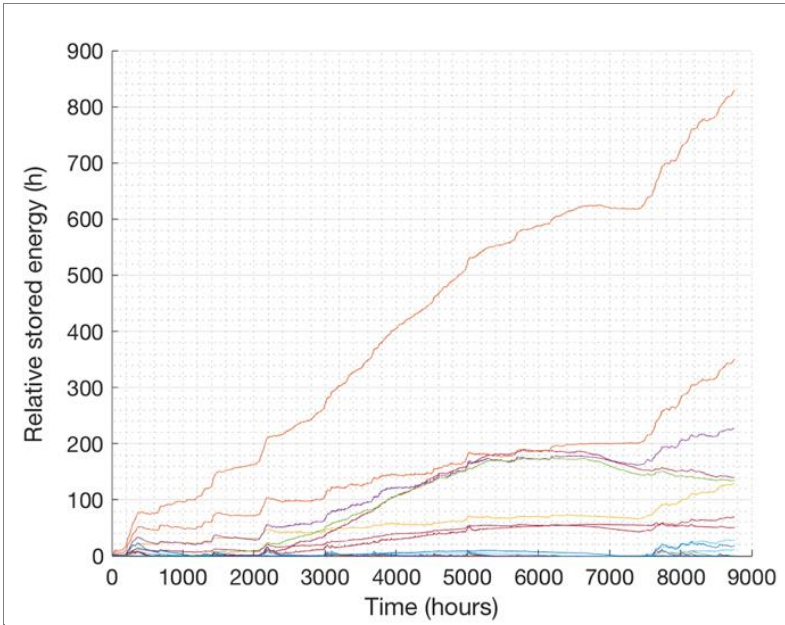

(a)

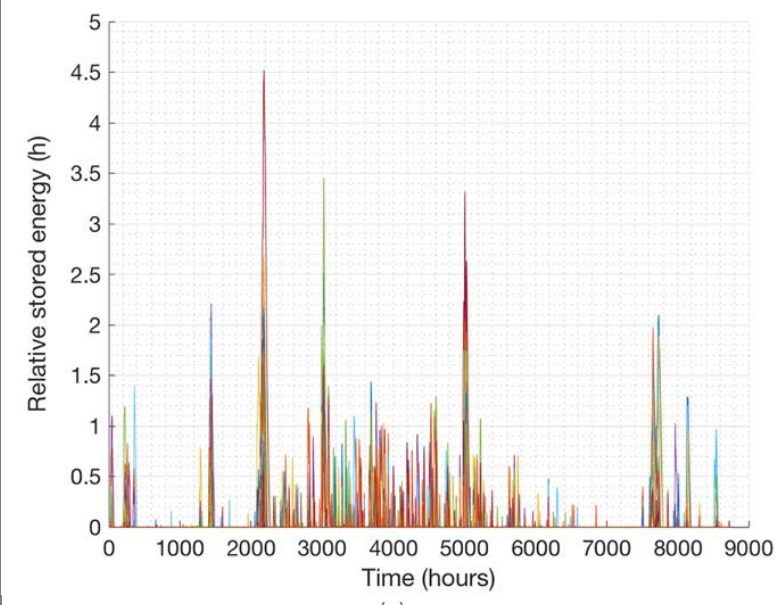

(c)

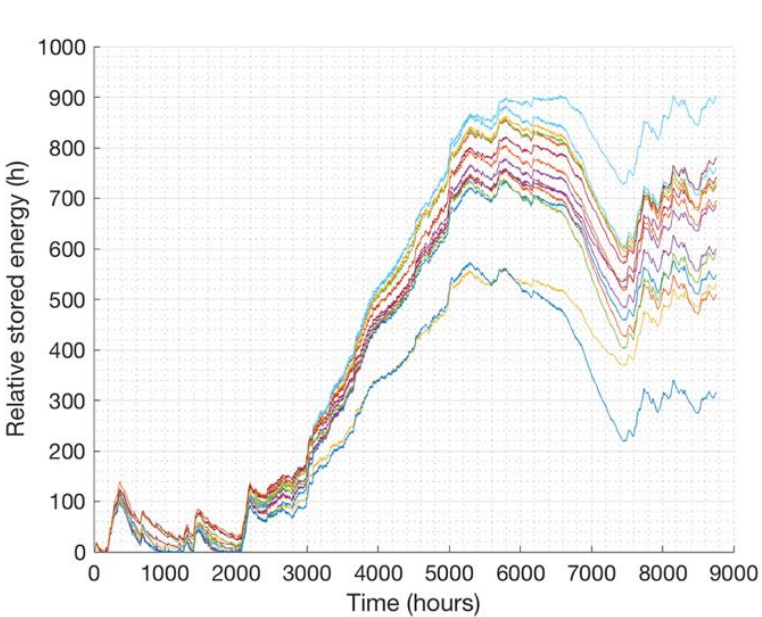

(b)

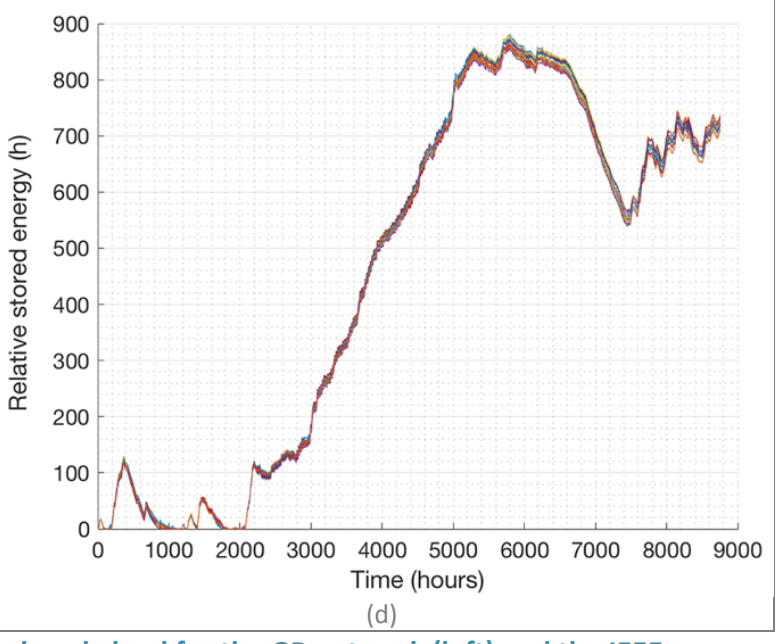

Figure 13: Hourly total stored energy relative to the maximum hourly load for the GB network (left) and the IEEE network (right) including renewable DGs with decentralised storage, where for each of the $\mathbf{3 0}$ simulation sets a curve has been plotted for the final decentralisation level (DL 9), each simulating a year for a CCF of $0 \%$. Results are shown for the random approach (a \& b) and strategy 3 (c \& d).

From the large variations in Figure 13a, it becomes clear that the locations of the DGs with storage matter for the amount of stored energy which is used in the network. If DGs are located in a part of the network which is unable to distribute the full amount of power generated by these DGs, the surplus power will be stored and the stored energy will accumulate. In contrast, when the DGs are linked to better connected nodes, the level of storage remains relatively low, as can be noticed in Figure 13c for most of the simulated hours in that particular case. The peaks in Figure $13 \mathrm{c}$ indicate that there are moments in which the total stored energy is multiple times larger than the maximum hourly load in the network, but that the stored energy will be used quickly afterwards, so that the accumulated total amount of energy storage will become zero again.

The results of the IEEE network appear to be different. Although the random approach (Figure 13b) also leads to large variations, none of the simulation sets shows a relatively low level of storage at the end of the year. This indicates that the locations of the DGs do not allow to optimally make use of the energy supplied by the DGs. From Figure 13d, strategy 3 applied to the IEEE network appears to result in neither a low level of storage at the end of the year, nor an overall profile of lower levels of storage. 


\subsubsection{Reserve capacity usage}

While reliability is an important aspect, the use of the reserve capacity is also an important performance indicator. An increased use of the reserve capacity will mean that more fossil fuel based plants are used and thus that less renewable energy (either direct or via energy storage) has been used. Moreover, in reality, the use of reserve capacity is often expensive. Figure 14 shows the average relative reserve capacity usage (RRCU) and the maximum RRCU per strategy for both networks. The average RRCU is defined as the ratio of the actual power supplied by the generators representing this reserve capacity to the total capacity available from these generators. The maximum RRCU is the maximum ratio found during each of the simulations sets.

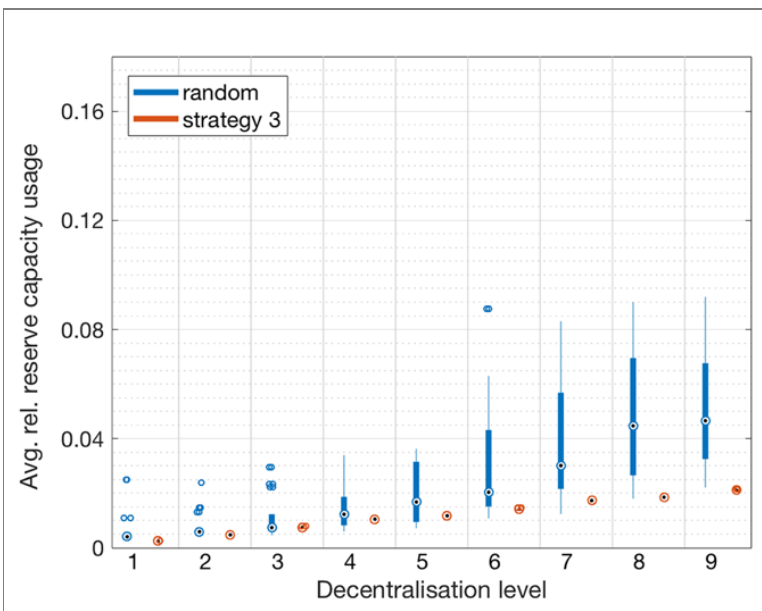

(a)

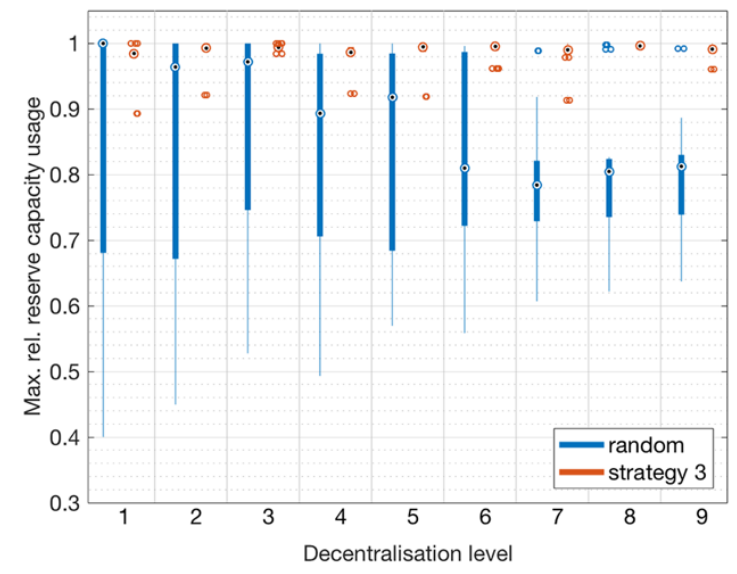

(c)

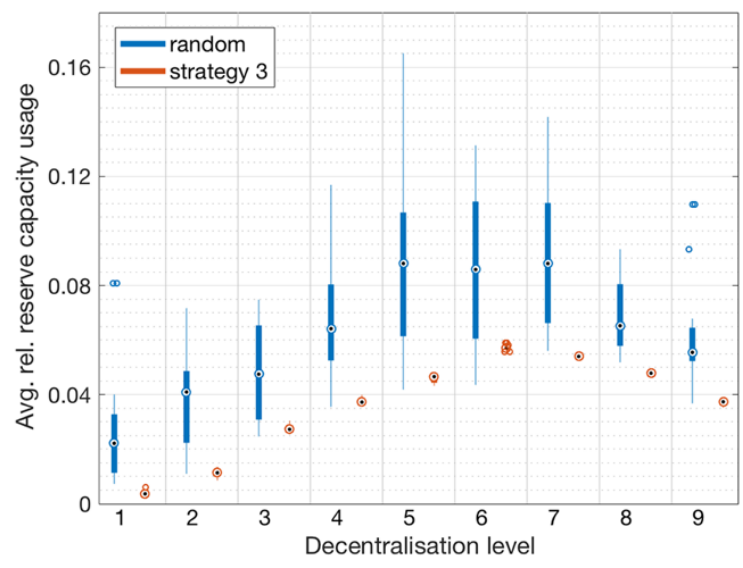

(b)

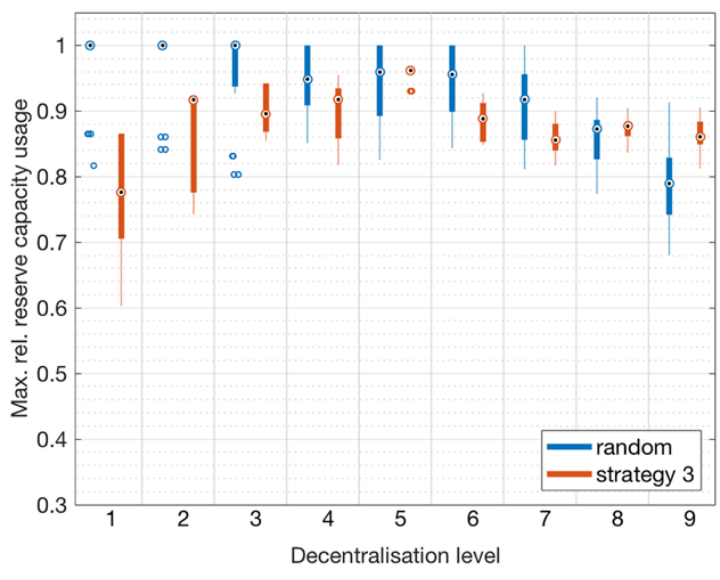

(d)

Figure 14: Average relative reserve capacity usage and maximum relative reserve capacity usage for different strategies for the GB network (left) IEEE network (right) including renewable DGs with decentralised storage, based on 30 simulation sets, each simulating a year with a time step of an hour for a CCF of $0 \%$.

From Figure 14 it can be observed that strategies 2 and 3 reduce the average usage of the reserve capacity for the GB network. However, looking at the maximum relative usage, it is interesting to see that these strategies increase the maximum relative usage compared with the random approach and strategy 1, with strategy 3 being close to 1 (i.e. 100\%). This means that although strategy 3 reduces the annual energy need from the reserve capacity, there seems to be little potential to reduce the overall capacity of the reserve if the most demanding moments are to be served. This might be related to the less optimal locations of the DGs under the random approach and strategy 1 , which will result in less direct power usage from DGs and therefore more power will be stored. 
Subsequently, the extra storage capacity will lessen the need of power from the reserve capacity during the most demanding moments.

For the IEEE network, in general, the strategies 2 and 3 perform slightly better than the random approach and strategy 1 . However, the differences among the strategies are less clear than those seen for the GB network. Also, the maximum relative usage of the reserve capacity is lower compared with the GB network.

\subsubsection{Varying CCF}

Increasing the Capacity Credit Factor (CCF) from 0\% to $100 \%$ with steps of 10\%, Figure 15 shows the blackout impact for each $\mathrm{DL}$, for both networks applying strategy 3 .
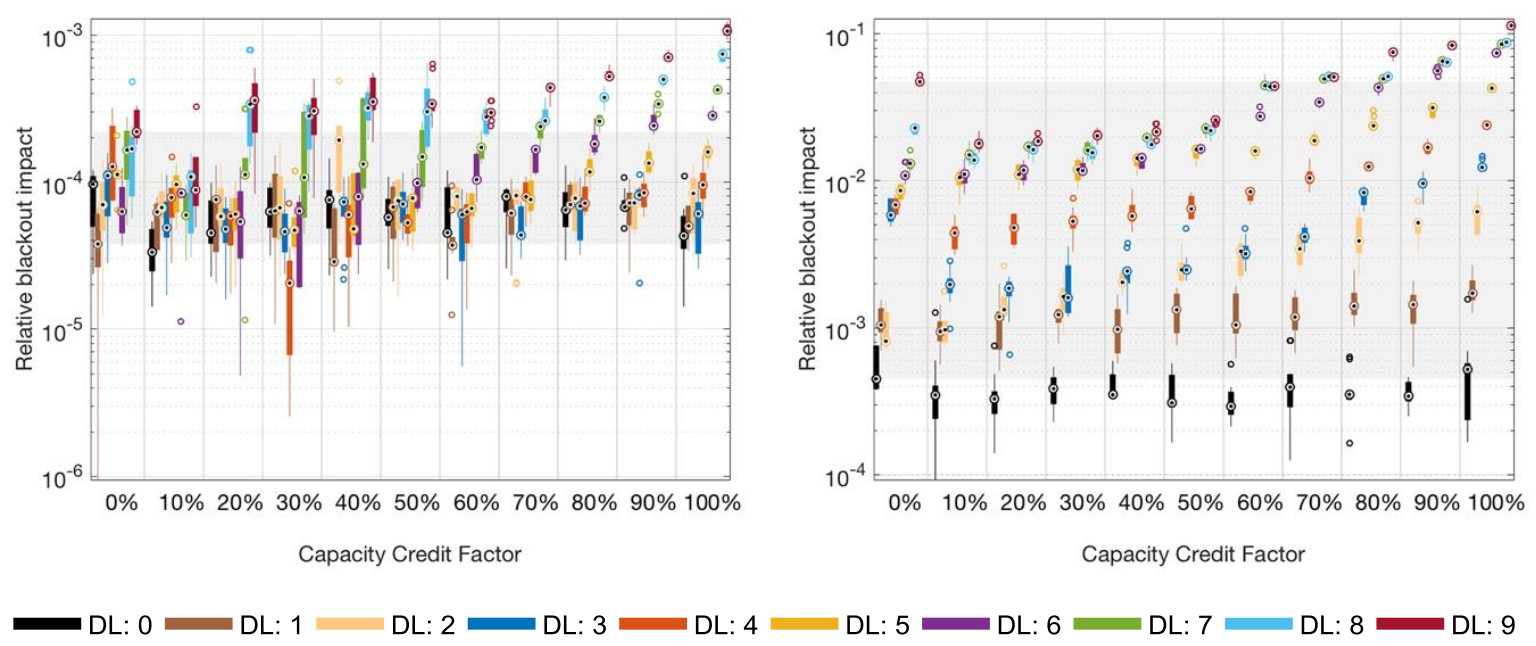

Figure 15: Blackout impact based on simulations with decentralised storage with strategy 3 for the GB network (left) IEEE network (right), based on ten simulation sets simulating a year with a time step of an hour for different DLs and CCFs.

The results in Figure 15 show that both networks become less reliable with increasing CCFs and become less reliable with increasing DLs. For CCF values in between $0 \%$ to $40 \%$, the relative blackout impact remains rather constant for the GB network. This means that keeping more than roughly $60 \%$ of the existing capacity online has little influence on the actual blackout impact. This stabilising effect is also visible for the IEEE network. Overall, the relative blackout impact for the IEEE network is larger compared with the GB network and therefore the IEEE network can be said to be less reliable than the GB network, which is in line with the previous findings.

Regarding the GB network, for a $100 \%$ CCF, a clear increasing trend is visible for increasing DLs as also observed for the IEEE network. However, for lower values of CCF, this trend becomes less clear and shows even a partly reversing trend for some DLs (e.g. 30\% CCF and DL 4). Furthermore, in the IEEE network, although the relative blackout impact generally increases with increasing values for $\mathrm{CCF}$, this does not hold for a CCF of $0 \%$. It has been difficult to identify the exact reasons for these irregularities, although they are generally considered to arise from the complex interactions between CCF, DL, optimal power flow determination, and network structure. 


\section{Discussion}

Overall, among the evaluated strategies to decentralise an existing network, strategy 3 , which takes both the generator impact as well as the power imbalance into account by selecting the generator to be replaced and the node to which the DG will be added, shows the best results. The decreased blackout impact and thus higher reliability can be attributed to an improved balance between demand and generation capacity across the network. The random strategy shows that if the power balance is not taken into account, the network becomes less reliable with an increasing level of decentralisation. This highlights an increasingly important role for the system operator and DNOs, and the value of improving their capability for network observation and management, in a decentralising system.

Applying DGs based on renewables with a varying power supply and a dynamic load profile shows similar results for the applied strategies as for the conventional DGs, in which the random approach generally gives the worst results and strategy 3 the best. However, the blackout impact increases with increasing levels of decentralisation, showing that both networks become less reliable. To a great extent, this can be explained by the assumptions made regarding the control mechanisms of the power output of the DGs. The assumption that the dispatch centre controls the amount of renewable power flowing into the grid and energy storage leads to a higher blackout impact when the dispatch centre does not function. In this case the total power output of a renewable DG has to be absorbed by the grid which leads to overloaded lines and subsequently causes line outages and eventually blackouts. Although in reality a variety of power controls would be present, these results show that the communication aspect of the electricity grid becomes crucial, and reinforces the previous point about the capabilities and roles for DNOs and the system operator. For a highly decentralised system with significant intermittent generation, completely new forms of grid management and regulation, with potentially different institutional arrangements may be warranted. This is outside the scope of the current paper, but is discussed in [48].

The relatively well-connected GB network mimics a real world HV network better than the IEEE network and would therefore be more illustrative of a real national system. Comparing the two networks, in general, the simulation results show that the GB network performs better than the IEEE network, which can be largely explained by the difference in network structure. The GB network is denser, has power supply and demand more evenly distributed over the network and has no node with one degree, whereas the IEEE network have nine nodes (all with a generator) with just one degree. Due to its higher density, the GB network has various options (i.e. different paths) for nodes to distribute or receive power. This is a network property which defines its reliability under stressed conditions (e.g. when a line outage occurs). It is clear that the network structure of the IEEE network has fewer options to (re)distribute power after specific lines will experience an outage. This is especially the case for large generators which are connected with a single line to the network. With a single line outage, these generators become isolated from the grid with no ability to transfer power to other nodes. Our simulation results suggest that a denser network structure, such as the GB network, is more reliable, which is also in line with findings presented in [22]; however, keeping this network structure while adding an increasing amount of DGs will need large additional investments in the infrastructure, because a more reliable network structure would require multiple power lines connected to a single DG. Other ways of achieving a higher reliability exist, such as those creating more flexibility in supply and demand (e.g. energy storage, demand side response). 
Since the techno-economic developments in technologies such as demand side response and energy storage technologies will have a strong impact on the economics of creating the extra flexibility in the grid, these developments will also determine whether it will be cost-effective to locate DGs in parts of the network where network constraints are currently not favourable. To find the optimal cost-effective solutions to ensure a reliable electricity supply with increasing amounts of DGs, it is therefore necessary to take a holistic view while keeping up-to-date with the latest developments in the field.

Some remarks regarding the setup of our study and some of its limitations will be discussed next. First, it should be noted that this study assesses the reliability effects of increased decentralisation of an electricity network by forcing it to operate near its critical point. Therefore, in reality, electricity grids are more reliable than one would argue based on the data presented in this study. Especially, overcapacity of both generators and power lines would increase the options to cope with line outages. However, the intention of this work has been to test the impact on reliability of different network topologies under extreme conditions, and thus relative performance is more important than absolute measures.

Furthermore, in this study it has been assumed that the power output of renewable-based DGs can only be curtailed by the dispatching centre. In practice, there is a variety of ways in which DGs can (partially) control their active power, for example, PV inverters normally operate in a specified voltage and frequency range and will disconnect otherwise. However, in the DC power flow model the voltage and grid frequency are not taken into account, therefore it is hard to model this power control behaviour. Hence, in reality the results will likely be in between the range of values obtained via the 'normal' model as applied in this study and the results which ensure that the dispatching centre always operates.

The assumption of unlimited energy storage is also a simplification of reality, although it is expected that this assumption would not have unduly influenced the results, since in this study most stored energy will be used relatively soon after it has been stored, as long as this is allowed by the network constraints.

The graphs showing the blackout impact, which are related to the network configuration including storage (Figure 15), should not be used to quantify the amount of reserve capacity required, because i) energy storage capacity is assumed to be limitless and ii) the simulations start at the beginning of a year with empty storage.

Regarding the usage of the reserve capacity as shown in Figure 14, there seems to be a clear tradeoff, which is related to the question: how to make optimal use of stored energy taking into account the need for reserve capacity? The cost of storage and reserve capacity will play a key factor in answering this question. Related to this is: who owns the storage and thus who has most interest in a specific cost strategy?

By replacing nine original generators with DGs, the IEEE network replaces $90 \%$ of its generators; for the GB network this is only $38 \%$. This different level of relative decentralisation of the network might also influence the results. 
The weather data applied in this study is based on a single year for a single location. In reality, the intermittency (e.g. consecutive days with low wind speed) will differ for each year and location. The different weather characteristics at different geographical locations will also have an influence on the power output of DGs, which will be more balanced in practice.

The applied time resolution of an hour might affect the specific results too, since a smaller time resolution might cause different supply and demand profiles potentially leading to different stresses in the network. However, the qualitative comparison between the outcomes of different strategies is not expected to alter significantly with the choice of time resolution.

In practice, a network operator has more options to increase the flexibility of the network, for example by using demand side response [49], which however has not been considered in this work. The same counts for measures such as network expansion and upgrading.

The central planner perspective applied in this study assumes that the government or network operator has control over where to install DGs. Although this seems unlikely to happen for distributed energy generators such as rooftop PV, for offshore wind parks this will be the case. Furthermore, in the future, network operators or governments could influence consumers who think of installing distributed systems by specific pricing or other policies.

Finally, this work has chosen HV networks to study the impact of (regionally) aggregated introduction of DGs, as explained in Section 2.1. Since most DGs would in reality be coupled to a LV network, lower level or multi-level network structures might reveal further details and characteristics of the effects of DGs on the reliability of a power network. Apart from the technical issues, the implementation of DGs requires interaction between the national and local energy systems. Ref [50] describes a methodology to assess how well local plans integrate in the larger national system. Such a methodology is important to align national and local energy planning.

\section{Conclusions}

This study set out to evaluate the impact of decentralisation on the reliability of electricity networks under stressed conditions.

The main findings are:

- Adding significant amounts of DGs, especially if they are intermittent, can seriously reduce network reliability; however, various approaches regarding the decentralisation strategy and management of the resulting network can mitigate the negative impact.

- Regarding network decentralisation with DGs based on conventional resources, the network becomes more reliable when the strategy improves the local power balance. In contrast, when arbitrary generators get replaced and DGs are connected to randomly chosen nodes, the network becomes less reliable. This could have implications for the design, regulations, or network connection charges for DGs in the future.

- Regarding network decentralisation with DGs based on renewables accompanied by decentralised energy storage, the results show that the network becomes less reliable regardless of the strategies applied, although strategy 2 and 3 perform generally better. Varying the CCF for different DLs shows that the networks become more reliable with 
decreasing CCFs and become less reliable with increasing DL. Keeping more than roughly $60 \%$ of the existing capacity online has little influence on the actual blackout impact.

However, the reliability can be greatly improved if the dispatch of generators is coordinated well, which leads to the last point:

- Communication between the DG and the electricity network and effective power control mechanisms are shown to be crucial for a more reliable power supply when there is an increased penetration of DGs based on renewables.

Based on the discussed limitations of this study, we recommend that future studies could enhance this work by focusing on the following points:

- Including a larger set of weather data. This will better resemble reality and will probably even out some of the electricity supply peaks, since wind speeds and solar irradiance would often vary from location to location.

- Running the model for different loading levels. This study assumed critical loading levels, in reality there is a capacity margin which allows the network to operate away from the critical levels, giving more room in the network for power re-routing.

- Changing the role of energy storage. Firstly, changing the merit order will influence the availability of stored energy during moments of low electricity supply by renewables and might lead to different results. Secondly, instead of co-locating energy storage, centralised energy storage can give other loading profiles in the network with potential benefits. Lastly, limiting the energy storage capacity could introduce additional constraints in the case of power surpluses in the network, which has not been considered in this work.

- Improving the power control mechanism to match the reality more closely. In reality, most DGs will have their own protection mechanisms and do therefore rely less on a central dispatching centre. Including this aspect will likely require the use of an AC power flow model, since the frequency and voltage levels will play a role for this.

- Including costs for different types of generators, energy storage, line additions etc., in order to quantify the cost of different strategies and relate them to the potential costs of the likely blackouts of a particular system.

\section{Literature}

[1] UK Government. Monthly feed-in tariff commissioned installations 2017. https://www.gov.uk/government/statistics/monthly-small-scale-renewable-deployment (accessed December 1, 2017).

[2] Amin SM, Wollenberg BFF. Toward a smart grid: power delivery for the 21st century. IEEE Power Energy Mag 2005;3:34-41. doi:10.1109/MPAE.2005.1507024.

[3] Li F, Qiao W, Sun H, Wan H, Wang J, Xia Y, et al. Smart transmission grid: Vision and framework. IEEE Trans Smart Grid 2010;1:168-77. doi:10.1109/TSG.2010.2053726.

[4] Ackermann T, Andersson G, Söder L. Distributed generation: A definition. Electr Power Syst Res 2001;57:195-204. doi:10.1016/S0378-7796(01)00101-8.

[5] Reza M, Schavemaker PH, Slootweg JG, Kling WL, Sluis L Van Der. Impacts of distributed generation penetration levels on power systems transient stability. IEEE Power Eng Soc Gen Meet 2004 2004;3115278118:1-6. doi:10.1109/PES.2004.1373261. 
[6] United Nations. The Paris Agreement 2016.

http://unfccc.int/paris_agreement/items/9485.php.

[7] Rinaldi SM, Peerenboom JP, Kelly TK. Identifying, understanding, and analyzing critical infrastructure interdependencies. IEEE Control Syst Mag 2001;21:11-25. doi:10.1109/37.969131.

[8] Kjølle GH, Utne IB, Gjerde O. Risk analysis of critical infrastructures emphasizing electricity supply and interdependencies. Reliab. Eng. Syst. Saf., vol. 105, 2012, p. 80-9. doi:10.1016/j.ress.2012.02.006.

[9] Matthewman S, Byrd H. Blackouts: a sociology of electrical power failure. Soc Sp (Przestrzeń Społeczna ... 2014:1-25.

[10] Hajiesmaili MH, Cai D, Mallada E. Understanding the Inefficiency of Security-Constrained Economic Dispatch. arXiv Prepr arXiv170600722 2017.

[11] Alsac O, Stott B. Optimal Load Flow with Steady-State Security. IEEE Trans Power Appar Syst 1974;PAS-93:745-51. doi:10.1109/TPAS.1974.293972.

[12] Aoki K, Satoh T. Economic dispatch with network security constraints using parametric quadratic programming. IEEE Trans Power Appar Syst 1982;PAS-101:4548-56. doi:10.1109/TPAS.1982.317308.

[13] Jabr R a., Coonick AH. A Homogeneous Linear Programming Algorithm for the Security Constrained Economic Dispatch Problem. IEEE Trans Power Syst 2000;15:930. doi:Article.

[14] Hedman KW, Ferris MC, O'Neill RP, Fisher EB, Oren SS. Co-optimization of generation unit commitment and transmission switching with $\mathrm{N}-1$ reliability. IEEE Trans Power Syst 2010;25:1052-63. doi:10.1109/TPWRS.2009.2037232.

[15] Ren H, Dobson I, Carreras BA. Long-term effect of the $n-1$ criterion on cascading line outages in an evolving power transmission grid. IEEE Trans Power Syst 2008;23:1217-25. doi:10.1109/TPWRS.2008.926417.

[16] Hemmati R, Hooshmand R-A, Khodabakhshian A. State-of-the-art of transmission expansion planning: Comprehensive review. Renew Sustain Energy Rev 2013;23:312-9. doi:10.1016/j.rser.2013.03.015.

[17] Moeini-Aghtaie M, Abbaspour A, Fotuhi-Firuzabad M. Incorporating large-scale distant wind farms in probabilistic transmission expansion planning-part II: Case studies. IEEE Trans Power Syst 2012;27:1594-601. doi:10.1109/TPWRS.2011.2182364.

[18] Lumbreras $S$, Ramos A. The new challenges to transmission expansion planning. Survey of recent practice and literature review. Electr Power Syst Res 2016;134:19-29. doi:10.1016/j.epsr.2015.10.013.

[19] Ofgem. Statutory Security of Supply Report 2016. 2016.

[20] Ward A. UK grid loses half the power from link to France. Financ Times Ltd 2016. https://www.ft.com/content/52e957a6-b64a-11e6-ba85-95d1533d9a62 (accessed November 1, 2017).

[21] Newmark Z. Apache helicopter crash causes power outage in Netherlands. NLTimes.nl 2017. https://nltimes.nl/2017/11/13/apache-helicopter-crash-causes-power-outage-netherlands (accessed July 20, 2011).

[22] Koç Y, Warnier M, Kooij RE, Brazier FMT. An entropy-based metric to quantify the robustness of power grids against cascading failures. Saf Sci 2013;59:126-34. doi:10.1016/j.ssci.2013.05.006.

[23] Koç $\mathrm{Y}$, Warnier $M$, Mieghem $P$ Van. The impact of the topology on cascading failures in electric power grids. arXiv Prepr arXiv ... 2013;31:1-19. doi:10.1016/j.physa.2014.01.056.

[24] Rohden $M$, Sorge A, Timme M, Witthaut D. Self-organized synchronization in decentralized power grids. Phys Rev Lett 2012;109. doi:10.1103/PhysRevLett.109.064101.

[25] Zerriffi H, Dowlatabadi H, Farrell A. Incorporating stress in electric power systems reliability models. Energy Policy 2007;35:61-75. doi:10.1016/j.enpol.2005.10.007.

[26] Carreras BA, Lynch VE, Sachtjen ML, Dobson I, Newman DE. Modeling blackout dynamics in 
power transmission networks with simple structure. hicss, vol. 1, 2001, p. 2018.

[27] Mei S, He F, Zhang X, Wu S, Wang G. An improved OPA model and blackout risk assessment. IEEE Trans Power Syst 2009;24:814-23. doi:10.1109/TPWRS.2009.2016521.

[28] Koonce AM, Apostolakis GE, Cook BK. Bulk power risk analysis: Ranking infrastructure elements according to their risk significance. Int J Electr Power Energy Syst 2008;30:169-83. doi:10.1016/j.ijepes.2007.06.013.

[29] Purchala K, Meeus L, Van Dommelen D, Belmans R. Usefulness of DC Power Flow for Active Power Flow Analysis. Power Eng Soc Gen Meet 2005:454-9. doi:10.1109/PES.2005.1489581.

[30] Zimmerman RD, Murillo-Sanchez CE, Thomas RJ. MATPOWER: Steady-State Operations, Planning, and Analysis Tools for Power Systems Research and Education. IEEE Trans Power Syst 2011;26:12-9. doi:10.1109/TPWRS.2010.2051168.

[31] McCarthy RW, Ogden JM, Sperling D. Assessing reliability in energy supply systems. Energy Policy 2007;35:2151-62. doi:10.1016/j.enpol.2006.06.016.

[32] Kueck JD. Measurement practices for reliability and power quality. 2005.

[33] Billinton R, Allan RN. Power-system reliability in perspective. Electron Power 1984;30:231-6. doi:10.1049/ep.1984.0118.

[34] Castro M. Assessing the risk profile to security of supply in the electricity market of Great Britain. Energy Policy 2017;111:148-56. doi:10.1016/j.enpol.2017.09.031.

[35] Merkel E, McKenna R, Fichtner W. Optimisation of the capacity and the dispatch of decentralised micro-CHP systems: A case study for the UK. Appl Energy 2015;140:120-34. doi:10.1016/j.apenergy.2014.11.036.

[36] UK Government. Quarterly and annual load factors 2017. https://www.gov.uk/government/publications/quarterly-and-annual-load-factors (accessed December 1, 2017).

[37] Castillo A, Gayme DF. Grid-scale energy storage applications in renewable energy integration: A survey. Energy Convers Manag 2014;87:885-94. doi:10.1016/j.enconman.2014.07.063.

[38] Ghofrani M, Arabali A, Etezadi-Amoli M, Fadali MS. Energy storage application for performance enhancement of wind integration. IEEE Trans Power Syst 2013;28:4803-11. doi:10.1109/TPWRS.2013.2274076.

[39] Denholm P, Sioshansi R. The value of compressed air energy storage with wind in transmission-constrained electric power systems. Energy Policy 2009;37:3149-58. doi:10.1016/j.enpol.2009.04.002.

[40] Beccali M, Brunone S, Finocchiaro P, Galletto JM. Method for size optimisation of large windhydrogen systems with high penetration on power grids. Appl Energy 2013;102:534-44. doi:10.1016/j.apenergy.2012.08.037.

[41] Bose S, Gayme DF, Topcu U, Chandy KM. Optimal placement of energy storage in the grid. Proc. IEEE Conf. Decis. Control, 2012, p. 5605-12. doi:10.1109/CDC.2012.6426113.

[42] Denholm P, Ela E, Kirby B, Milligan M. The role of energy storage with renewable electricity generation 2010.

[43] Aho J, Buckspan A, Laks J, Fleming P, Jeong Y, Dunne F, et al. A tutorial of wind turbine control for supporting grid frequency through active power control. Am. Control Conf. (ACC), 2012, IEEE; 2012, p. 3120-31.

[44] Bukhsh W, McKinnon K. Network data of real transmission networks. Publ Online http//www.maths.ed.ac.uk/optenergy/NetworkData/ 2013.

[45] Bell KRW. Test system requirements for modelling future power systems. IEEE PES Gen. Meet., IEEE; 2010, p. 1-8.

[46] Newman M. Networks. An introduction. 2010. doi:10.1093/acprof:oso/9780199206650.001.0001.

[47] Latora V, Marchiori M. Efficient behaviour of small-world networks. Phys Rev Lett 2001;87:198701 (pp. 4). doi:10.1103/PhysRevLett.87.198701.

[48] Barton J, Emmanuel-Yusuf D, Hall S, Johnson V, Longhurst N, O'Grady A, et al. Distributing 
Power. A transition to a civic energy future 2015.

[49] National Grid. Balancing services 2017.

https://www.nationalgrid.com/uk/electricity/balancing-services (accessed December 1, 2017).

[50] Thellufsen JZ, Lund $\mathrm{H}$. Roles of local and national energy systems in the integration of renewable energy. Appl Energy 2016;183:419-29. doi:10.1016/j.apenergy.2016.09.005. 


\section{A. Results of all strategies}

\section{Part I: DGs based on conventional sources}

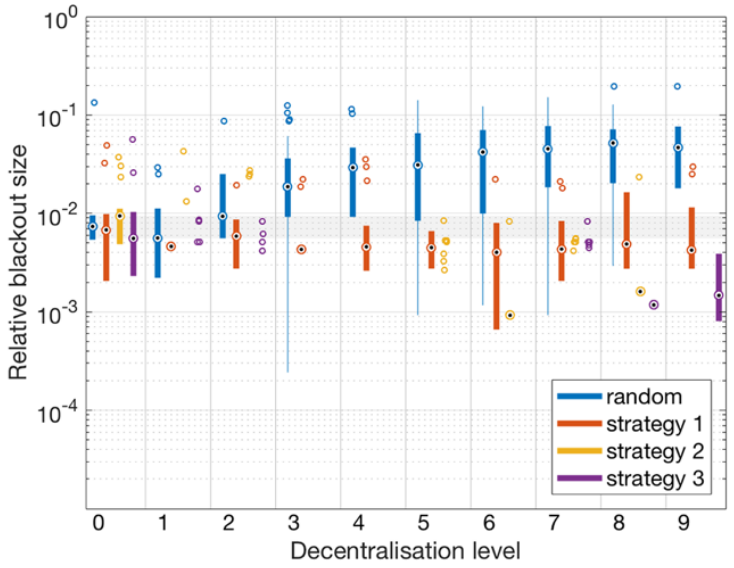

(a)

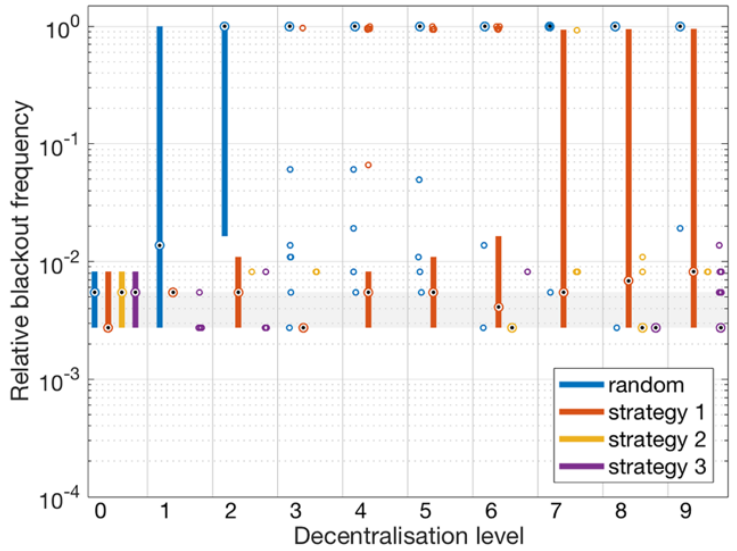

(c)

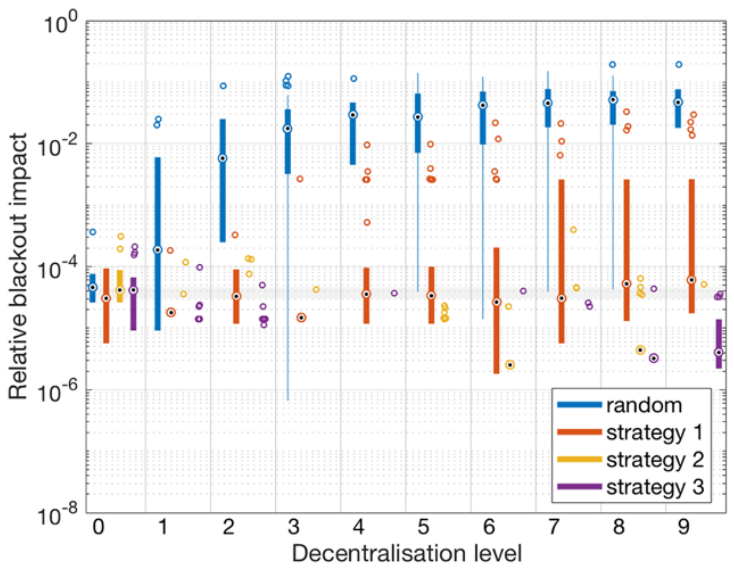

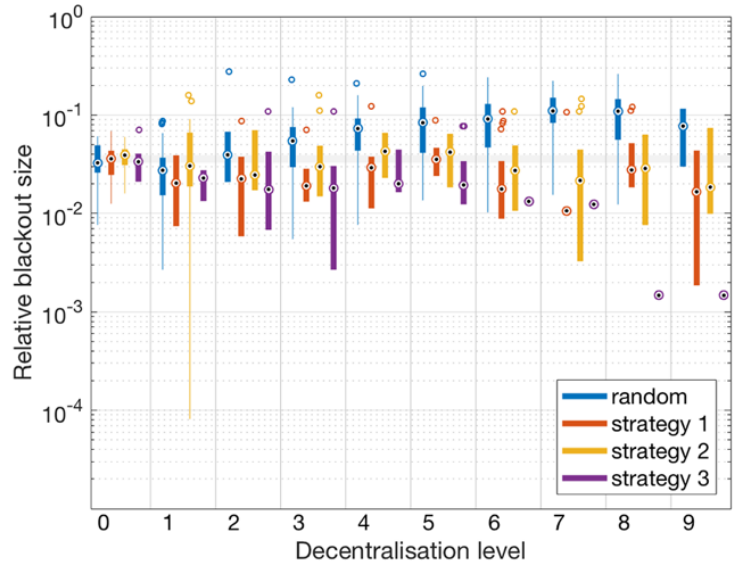

(b)

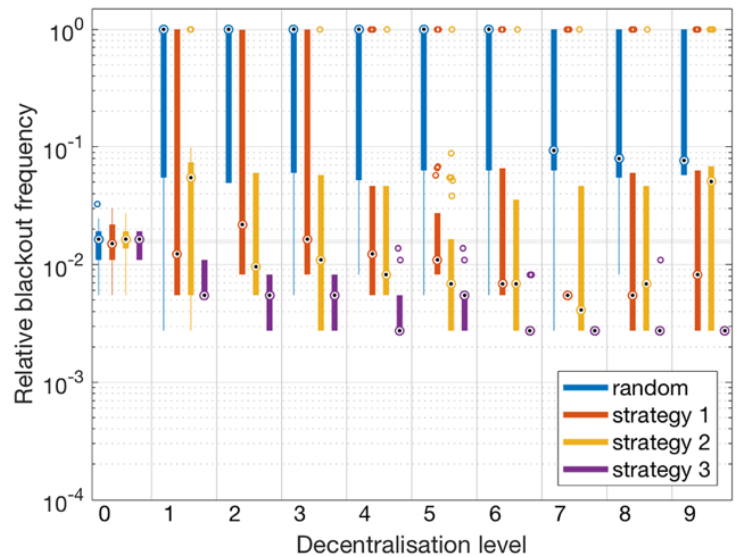

(d)

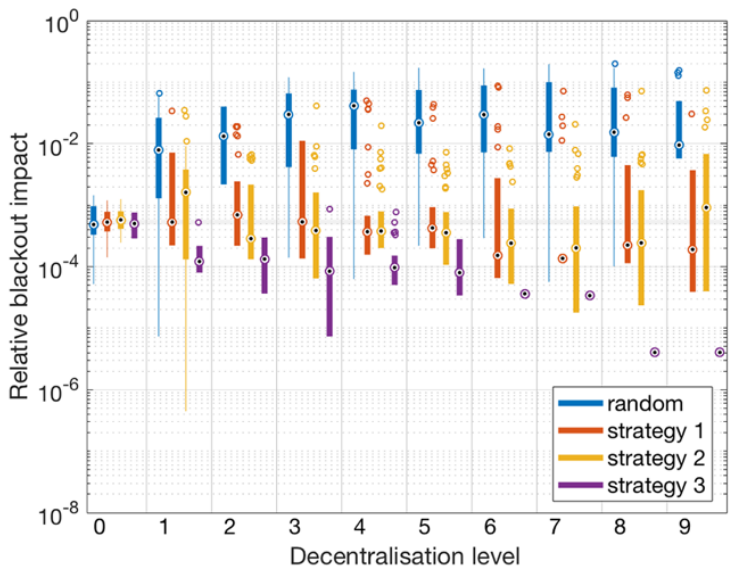

(f)

Figure 16: Relative blackout size (a, b), relative blackout frequency $(c, d)$ and relative blackout impact (e, f) for the reduced GB network (left) and the IEEE 39 network (right) with conventional DGs, based on 30 simulation sets, each simulating a year with a time step of a day, for different levels of decentralisation, for all strategies. 


\section{Part II: DGs based on renewables}

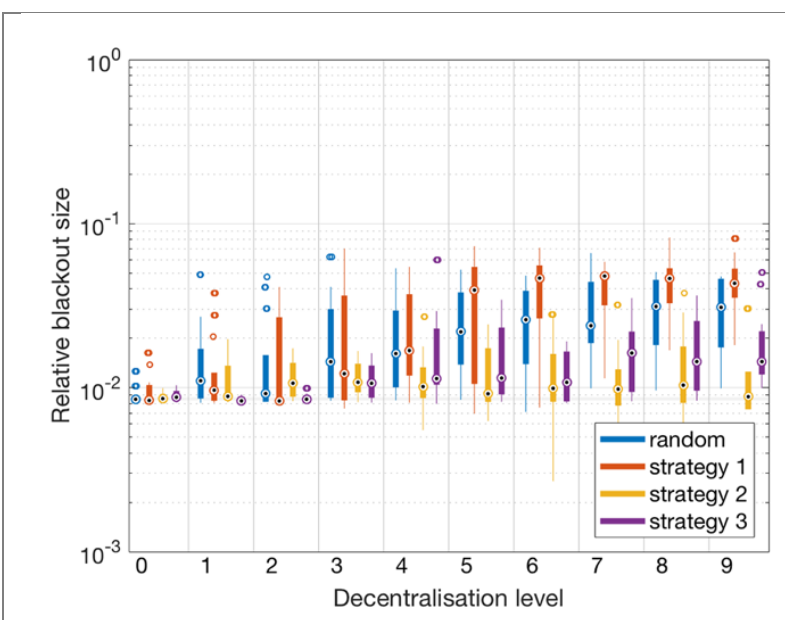

(a)

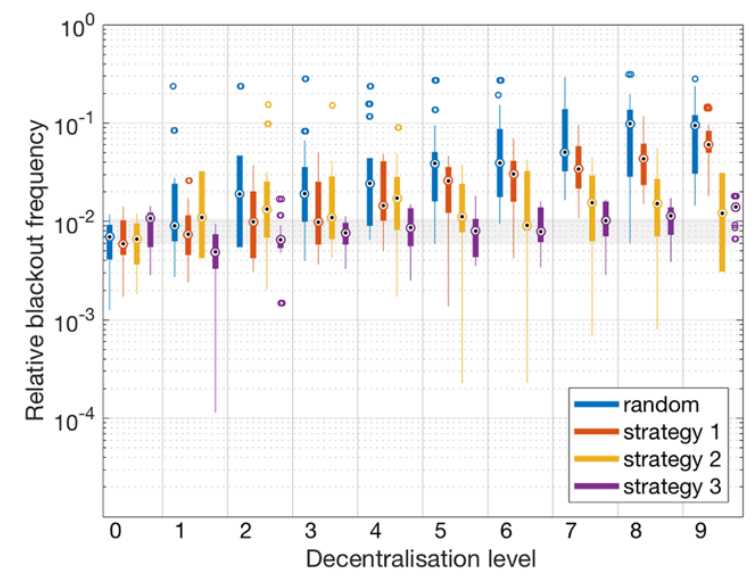

(c)

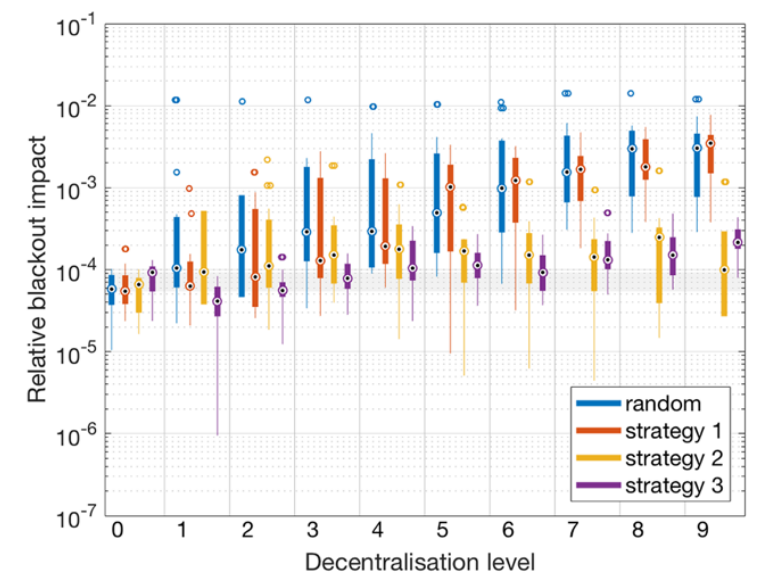

(e)

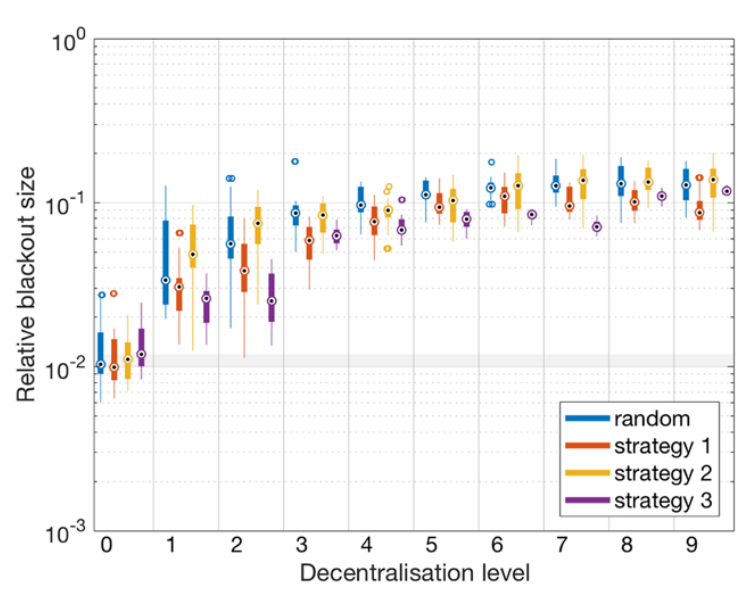

(b)

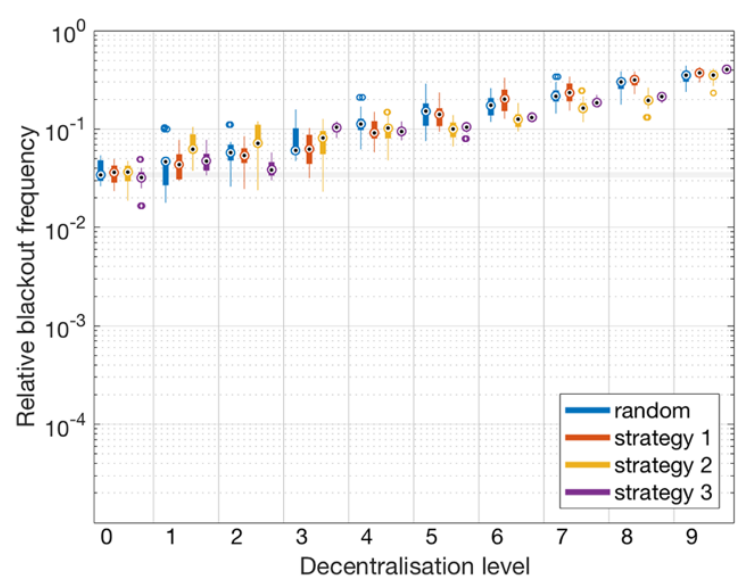

(d)

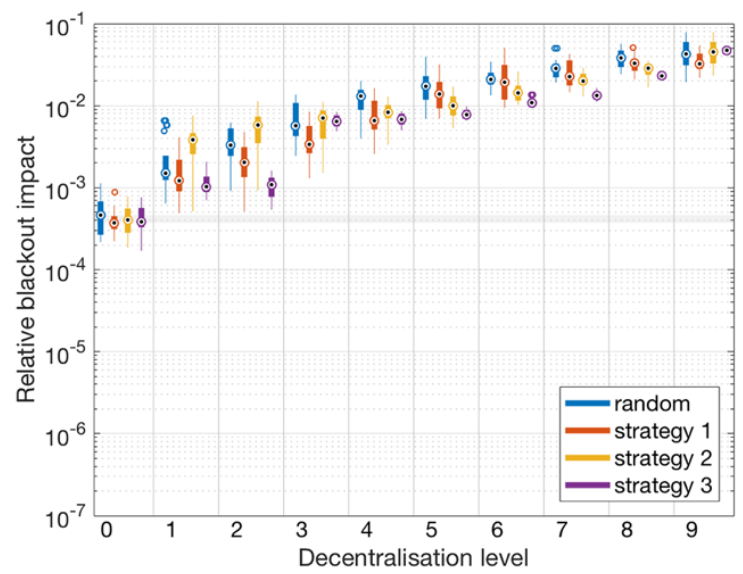

(f)

Figure 17: Relative blackout size (a, b), relative blackout frequency (c, d) and relative blackout impact (e, f) for the reduced GB network (left) and the IEEE 39 network (right) including renewable DGs with decentralised storage, based on $\mathbf{3 0}$ simulation sets, each simulating a year with a time step of an hour, for different decentralisation levels for a CCF of $0 \%$, for all strategies. 


\section{B. Generator impact}

In this appendix the impact of each generator on the network will be studied. For these simulations, the random blackout occurrence has been excluded; however, the probabilities of a line getting tripped and the probability of a non-functioning dispatching centre have been kept the same. All loads are multiplied by a constant factor, such that the total load equals a percentage of the total generation capacity (i.e. a certain loading level will be achieved).

The procedure to determine the generator impact is as follows: For each of the generators, the generation capacity will be moved to each of the nodes in the network, one at a time. For each combination, the power flow model will be run for 100 days with a time step of a day and the relative blackout impact will be determined. The generators are assumed to be based on conventional generators, so the generation capacity remains constant. If a generator/node combination results in a blackout, this combination will be checked during the next simulation at a $5 \%$ lower loading level until no blackouts occur.

The analysis shows that the GB network is quite unbalanced. Moving any capacity to node 1 or 2 will result in a blackout. Moreover, looking at the network structure as shown in Figure 3, it is clear that the lines connecting the nodes in the lower region (e.g. nodes 1 to 7 ) have a lower capacity. So, surplus power in this region experiences difficulties to distribute the power to the higher region. This effect is exaggerated by generator at node 16 which has the largest generation capacity. Comparing the maximum relative blackout impact with the maximum generation capacity per generator, as shown in Figure 18, shows that there is a strong correlation between these two quantities $(r=0.995)$.

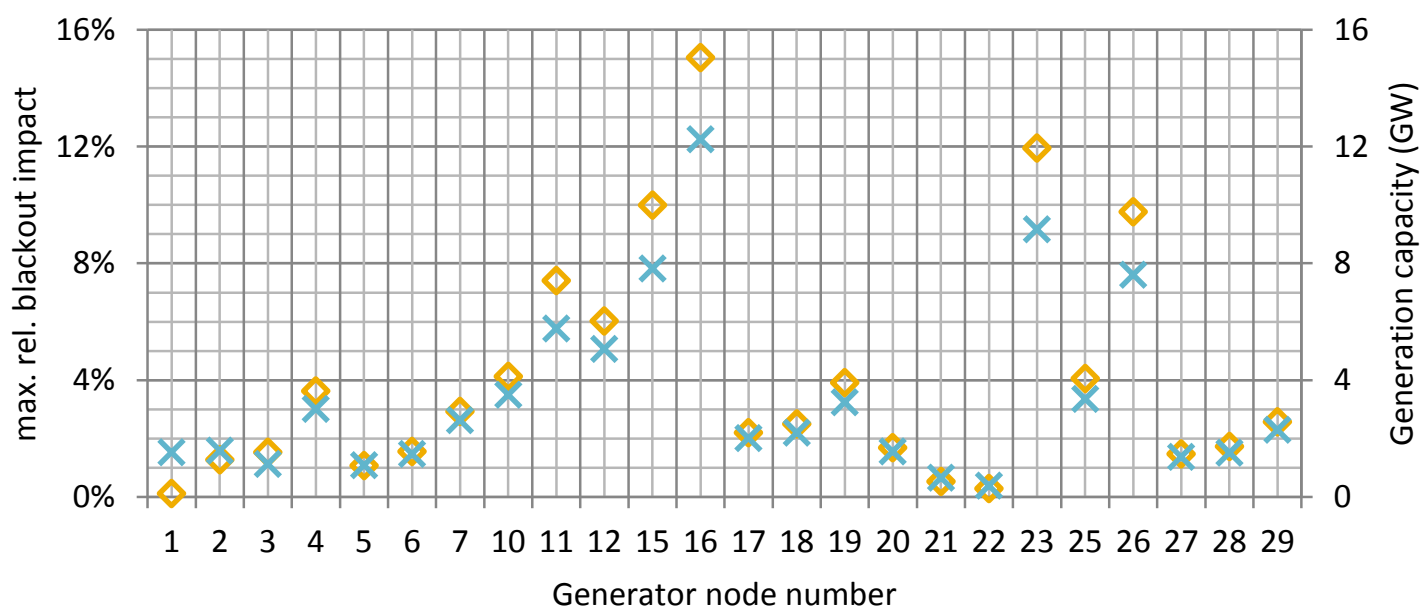

$\checkmark$ max rel. blackout impact $\times$ Max. generation capacity

Figure 18: The maximum relative blackout impact compared with the maximum generation capacity per generator for the GB network.

Some maximum blackout impacts deviate slightly more from their corresponding maximum generation capacity than others. Other factors would also play a role. For instance, node 16 has the highest degree and second highest betweenness, so its impact on the relative blackout size could be strengthened by this. The same counts for node 15 , which has the highest betweenness and a high 
degree. Generators at nodes 23 and 26 do have similar characteristics, with generation capacities of roughly $9 \mathrm{GW}$ and $8 \mathrm{GW}$, respectively, although their degree and betweenness are around average. Node 11 has a degree of 4 , a betweenness of 91 and a relative generation capacity of $7 \%$. Location of the generators and loads in the network and the line capacities will also play a role.

Table 5: Top 5 of node numbers based on various metrics for the GB network

\begin{tabular}{|r|r|r|r|r|r|}
\hline Rank & Impact & Pmax & Load & Degree & Betweenness \\
\hline 1 & 16 & 16 & 25 & 16 & 15 \\
\hline 2 & 23 & 23 & 18 & 4 & 16 \\
\hline 3 & 15 & 15 & 23 & 13 & 9 \\
\hline 4 & 26 & 26 & 11 & 15 & 6 \\
\hline 5 & 11 & 11 & 28 & 21 & 11 \\
\hline
\end{tabular}

Table 5 shows the top 5 of node numbers based on various metrics. Comparing the node numbers with the impact per bus confirms that the maximum generation capacity is an important measure for the relative blackout size.

Figure 19 compares the maximum relative blackout impact with the maximum generation capacity per generator for the IEEE network. The correlation is clearly visible again, but is less strong $(r=0.662)$.

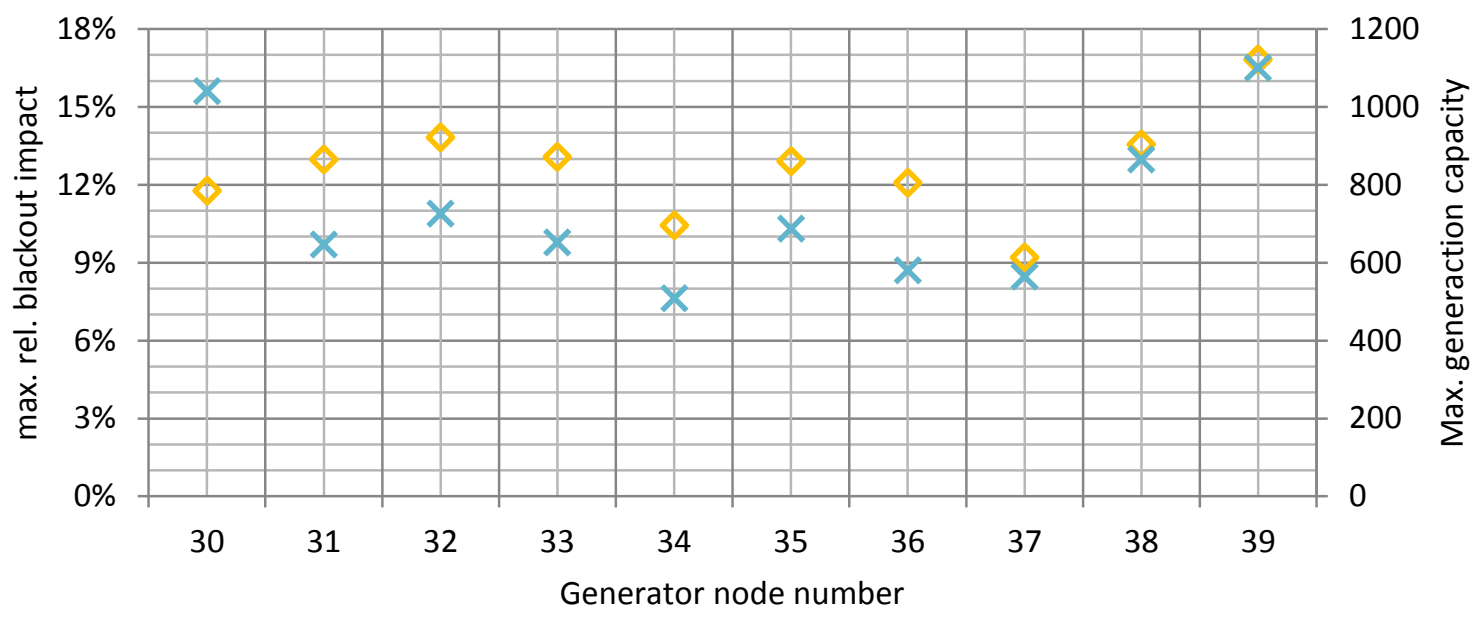

$\diamond$ max rel. blackout impact $\quad \times$ Max. generation capacity

Figure 19: The maximum relative blackout impact compared with the maximum generation capacity per generator for the IEEE network.

Table 6 shows the node numbers which are in the top 5 based on various metrics. The correlation between generator impact and maximum generator capacity is less strong compared with the results obtained for the GB network. 
Table 6: Top 5 of node numbers based on various metrics for the IEEE network

\begin{tabular}{|r|r|r|r|r|r|}
\hline Rank & Impact & Pmax & Load & Degree & Betweenness \\
\hline 1 & 39 & 39 & 39 & 16 & 16 \\
\hline 2 & 32 & 30 & 20 & 2 & 14 \\
\hline 3 & 38 & 38 & 8 & 26 & 4 \\
\hline 4 & 33 & 32 & 4 & 6 & 17 \\
\hline 5 & 31 & 35 & 16 & 14 & 3 \\
\hline
\end{tabular}

Another important difference between both networks, which can be observed by comparing Figure 18 with Figure 19, is that for the IEEE network, each generator results in a relative blackout impact in the range of $9.2 \%-16.8 \%$, whereas the GB network shows some generators with a significant lower impact and has a range of $0.1 \%-15.0 \%$. Moreover, when ordering the generator impacts of the $G B$ network in ascending order, the generator with the ninth lowest relative blackout impact has only a relative blackout impact of $1.7 \%$. Only from the $21^{\text {st }}$ generator onwards, a larger relative blackout impact of $9.8 \%$ is shown compared with the lowest one in the IEEE network. This might explain the differences in the effectiveness of the applied strategies to both networks. However, these differences in blackout impact are likely a result of the difference in the network topologies. 


\section{Unsolved Optimal Power Flows}

MATPOWER's Interior Point Solver has been applied in the optimal power flow (OPF) algorithm. When the algorithm does not converge fast enough, there is a chance that the OPF is not able to solve the linear equations within the maximum number of iterations. The latter has been set to 200 .

The two tables in this appendix show the amount of power flow runs in which the maximum number of iterations has been reached without a solution. The total number of power flow runs related to the simulations presented in Table 7 are 2,628,000 for each strategy (365 days x 24 hour $x 10$ DLs $x$ 30 simulations sets). For Table 8 this corresponds to 876,000 power flow runs for each network per CCF, as these results are based on ten simulation sets per CCF value. The exact reason behind this and why some strategies show more of these unsolved cases is yet to be found.

Table 7: Number of unsolved optimal power flows for various strategies for both networks related to Part II.

\begin{tabular}{|l|r|r|r|r|}
\hline \multicolumn{1}{|c|}{ Simulation name } & \multicolumn{1}{c|}{ Random } & \multicolumn{1}{c|}{ Strategy 1 } & \multicolumn{1}{c|}{ Strategy 2 } & \multicolumn{1}{c|}{ Strategy 3 } \\
\hline GB network in part II & 14 & 32 & 6 & 6 \\
\hline IEEE network in part II & 392 & 354 & 462 & 1,044 \\
\hline $\begin{array}{l}\text { GB network, OPF always } \\
\text { operating }\end{array}$ & 21 & 10 & 3 & 2 \\
\hline $\begin{array}{l}\text { IEEE network, OPF always } \\
\text { operating }\end{array}$ & 584 & 558 & 568 & 356 \\
\hline
\end{tabular}

Table 8: Number of unsolved optimal power flows for both networks with varying the CCF.

\begin{tabular}{|c|r|r|r|r|r|r|r|r|r|r|r|}
\hline CCF & $\mathbf{1 0 0 \%}$ & $\mathbf{9 0 \%}$ & $\mathbf{8 0 \%}$ & $\mathbf{7 0 \%}$ & $\mathbf{6 0 \%}$ & $\mathbf{5 0 \%}$ & $\mathbf{4 0 \%}$ & $\mathbf{3 0 \%}$ & $\mathbf{2 0 \%}$ & $\mathbf{1 0 \%}$ & $\mathbf{0 \%}$ \\
\hline GB network & 6 & 1 & 18 & $\mathbf{7}$ & 2 & 4 & 203 & 7 & 0 & 4 & 2 \\
\hline IEEE network & 809 & 104 & 200 & 410 & 310 & 116 & 160 & 231 & 258 & 260 & 379 \\
\hline
\end{tabular}

